UC-34

Issued: June 1987

LA--11001-MS

DE87 010838

\title{
CAVEAT Calculations of Shock Interactions
}

\author{
Donald L. Sandoval
}

\section{DISCLAIMER}

This report was prepared as an account of work sponsored by an agency of the United States Government. Neither the United States Government nor any agency thereof, nor any of their employees, makes any warranty, express or implied, or assumes any legal liability or responsibility for the accuracy, completeness, or usefulness of any information, apparatus, product, or process disclosed, or represents that its use would not infringe privately owned rights. Reference herein to any specific commercial product, process, or service by trade name, trademark, manufacturer, or otherwise does not necessarily constitute or imply its endorsement, recommendation, or favoring by the United States Government or any agency thereof. The views and opinions of authors expressed herein do not necessarily state or reflect those of the United States Government or any agency thereof. 


\section{CORTEITS}

I. INTRODUCTION

II. RIGID WEDGES $r$

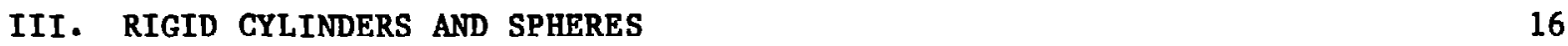

$\begin{array}{lr}\text { IV. DEFORMABLE CYLINDRICAL AND SPHERICAL SHELLS } & 28\end{array}$

$\begin{array}{lr}\text { V. DEFORMABLE CONICAL SHELLS } & 41\end{array}$

VI. QUESTIONS AND DISCREPANCIES $\quad 46$

A. Discrepancies of Figure $4 \quad 49$

B. Discrepancles of Figure $7 \quad 50$

C. Discrepancies of Figure 17

VII. CONCLUSION

$\begin{array}{lr}\text { ACKNOWLEDGMENTS } & 52\end{array}$

$\begin{array}{lr}\text { REFERENCES } & 53\end{array}$ 
CAVEAT CALCULATIONS OF SHOCK INTERACTIONS

by

Donald L. Sandoval

\begin{abstract}
CAVEAT is a computer code for calculating the time-varying fluid dynamics of several adjacent materials in two or three space dimensions. Using an extended Godunov technique and adaptive meshing, the code allows for large slippage at material interfaces. To exhiblt the capability for calculating strong distortions we have performed a varlety of calculations describing the interaction of shocks with rigid wedges, cylinders, and spheres and deformable cylindrical, spherical, and confical shells in two space dimensions. Comparison of the results with experimental data and analytical solutions demonstrates the considerable accuracy that can be expected from calculations with this code.
\end{abstract}

\title{
I. INTRODDCTION
}

For solutions of complex problems in the dynamics of fluids it is often necessary to employ numerical technlques, and to accomplish these solutions by means of high speed computers. This 1s espectally so for time varying problems in two or three space dimensions. With several materlals undergolng strong contortions the challenge 18 accentuated by the necessity for resolving slippage at the material Interfaces.

Numerous numerlcal techniques have been devised over the past 50 years to meet these challenges, but none have possessed the degree of generallty that would be desirable for solving the scope of problems generated by today's sclentific and englneering needs. To extend considerably our capability in the fleld of fluld dynamics, a new computer code called CAVEAT [1] has been developed over the last several years at this Laboratory. Some essentlal features of this numerical technique are: 
- the Lagrangian representation of material interfaces,

- cell centered velocities with arbitrary adaptivity of the finite difference mesh,

- a generalized Godunov solution procedure, and

o a general representation of material properties including equation of state and strength.

In this report our goal is to exhibit some of the features of the CAVEAT code. To achieve this goal we investigate the interaction of shocks in air with rigid wedges, cylinders, and spheres, and with deformable cylindrical, spherical, and conical shells. We make comparisons of computational results with theoretical and analytical calculations and also with experimental data. For example, we compare pressures on the surfaces of rigid objects with experimental results presented by Hellig and Reichenbach [2]. We also compare computer-generated depictions of the shock structure generated by the CAVEAT code to interferograms of similar experimental data presented by Ben-Dor and Glass [3]. We also Invest1gate shock Interaction with deformable cylinders and spheres and conical shells in which the density of the deformable object is large, to simulate an slmost rigid obstacle, or small, in order to demonstrate the capability for large deformations .

From these results we demonstrate the ability of the CAVEAT code to give an accurate description of the complex processes of shocks in gases interacting with rigid wedges, cylinders, and spheres, and with deformable cylindrical, spherical, and conical she11s. Table I defines the nomenclature that is used in these studies.

\section{RIGID WEDGES}

For the rigld wedge studies we are concerned with the reflected shock structure, and with the pressures generated by the shock interaction with the wedge surface as a function of the wedge angle for varlous incident-shock Mach numbers. Three types of shock configurations are to be expected:
- simple reflection,
o Mach reflection, and
- double Mach reflection.

We exhibit calculations for all three clrcumstances and also calculate the surface pressures generated by these shocks, which are compared with the experimental data of Hellig and Relchenbach. 
TABLE 1

\section{LIST OP PARAMBTERS}

$\theta_{w}$ - wedge angle

${ }^{\theta_{I}}$ - angle of Incident

$\gamma$ - parameter in polytropic gas equation of state

$\gamma_{\text {SH }}$ - parameter in polytropic gas equation of state for shell region

$c_{0}$ - Initial sound speed in the pre-shocked region

$I_{0}$ - Initial internal energy in the pre-shocked region

I_ - Initial energy in the post-shocked region

M - Incident-shock Mach number

$p_{o}$ - initial pressure in the pre-shocked region

P_ - initial pressure in the post-shocked region

$\mathrm{p}_{\mathrm{SH}}$ - initial pressure in the shell region

$\mathrm{p}_{1}$ - pressure behind the reflected and (or) primary Mach stem

$P_{2}$ - pressure behind the secondary Mach shock

$\rho_{0}$ - Iritial density in the pre-shocked region

$\rho_{\text {_ }}$ - Initial density in the post-shocked region

$\rho_{\text {SH }}$ - initial density in the shell region

$u_{\text {- }}$ - material velocity in the post-shocked region

$v_{s}-$ incident shock velocity 
Figure 1 shows our set-up configuration and initial parameters. The bottom 1s our prescribed Inflow boundary with the top boundary being outflow. The left and right boundaries are both reflective boundaries. Our pre-shocked material is always inftially at rest, and the input quantities are nondimensionalized in terms of initial sound speed, density, and pressure. Specifying the initial Mach number, $M$, to correspond with experimental values we calculate our post-shock conditions (i.e., our inflow conditions) using the following polytropic gas equations [4]:

$$
M=\frac{v_{s}}{c_{0}},
$$

$$
\frac{u_{-}}{c_{0}}=\frac{2\left(M^{2}-1\right)}{(\gamma+1) M} \text {, }
$$

$$
\frac{\rho_{-}}{\rho_{0}}=\frac{\gamma+1}{\gamma-1+\left(\frac{2}{M^{2}}\right)} \text {, }
$$

$$
\frac{p_{-}}{p_{0}}=1+\frac{2 \gamma}{\gamma+1}\left(M^{2}-1\right) .
$$

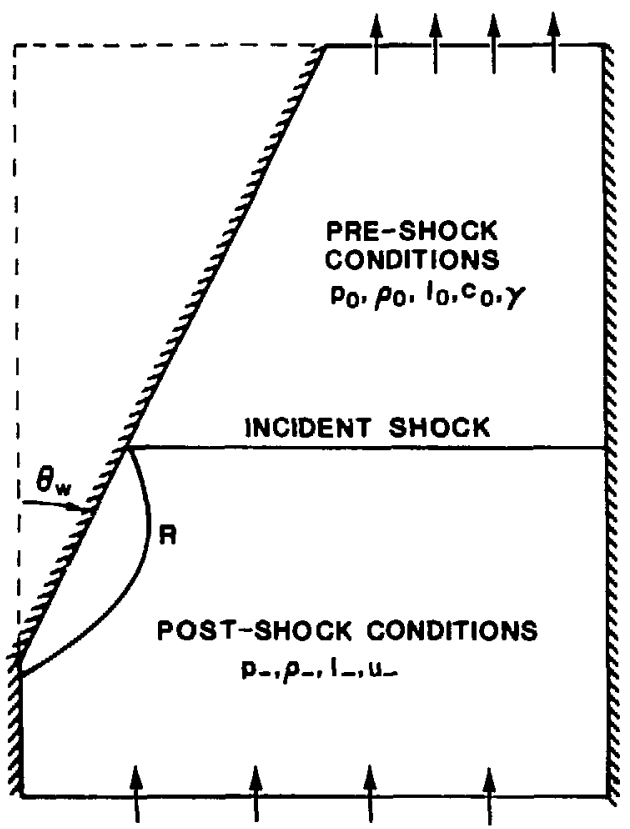

Fig. 1. Configuration for the wedge problems. The left and right boundaries are reflective. The top 18 outflow and the bottom inflow. $R$ is the reflected shock in this example. 
Table II lists the initial pre-shocked and post-shocked conditions calculated from the above equations along with problem ID number and the corresponding figure numbers.

TABLE II

\section{INPUT DATA FOR RIGID WEDGE CALCULATIONS}

\begin{tabular}{|c|c|c|c|c|c|c|c|c|c|c|c|c|c|}
\hline $\begin{array}{c}\text { figure } \\
\text { Number }\end{array}$ & $\begin{array}{c}\text { Run } \\
\text { Number }\end{array}$ & $M$ & $v_{s}$ & $\rho_{0}$ & $I_{0}$ & $p_{0}$ & $c_{0}$ & $Y$ & $\rho_{-}$ & $I_{-}$ & $p_{-}$ & $U_{-}$ & $\theta_{W}$ \\
\hline 2 & WDG30-1v & 3.36 & 3.36 & 1.0 & 1.786 & 0.714 & 1.0 & 1.4 & 4.16 & 5.58 & 9.29 & 2.55 & 30 \\
\hline 6 & WDG45-1v & 3.36 & 3.36 & 1.0 & 1.786 & 0.714 & 1.0 & 1.4 & 4.16 & 5.58 & 9.29 & 2.55 & 45 \\
\hline & WDG49-1v & 3.36 & 3.36 & 1.0 & 1.786 & 0.714 & 1.0 & 1.4 & 4.16 & 5.58 & 9.29 & 2.55 & 49 \\
\hline & WDG5--1v & 3.36 & 3.36 & 1.0 & 1.786 & 0.714 & 1.0 & 1.4 & 4.16 & 5.58 & 9.29 & 2.55 & 51 \\
\hline 5 & WDG55-1v & 3.36 & 3.36 & 1.0 & 1.786 & 0.714 & 1.0 & 1.4 & 4.16 & 5.58 & 9.29 & 2.55 & 55 \\
\hline 8.52 & WDG35-2v & 1.26 & 1.26 & 1.0 & 1.786 & 0.714 & 1.0 & 1.4 & 1.45 & 2.08 & 1.2 & 0.389 & 35 \\
\hline 8 & WDG44-2v & 1.26 & 1.26 & 1.0 & 1.786 & 0.714 & 1.0 & 1.4 & 1.45 & 2.08 & 1.2 & 0.389 & 44 \\
\hline & WDG45-2v & 1.26 & 1.26 & 1.0 & 1.786 & 0.714 & 1.0 & 1.4 & 1.45 & 2.08 & 1.2 & 0.389 & 45 \\
\hline 8.52 & WDG47-2v & 1.26 & 1.26 & 1.0 & 1.786 & 0.714 & 1.0 & 1.4 & 1.45 & 2.08 & 1.2 & 0.389 & 47 \\
\hline & WDG55-2v & 1.26 & 1.26 & 1.0 & 1.786 & 0.714 & 1.0 & 1.4 & 1.45 & 2.08 & 1.2 & 0.389 & 55 \\
\hline 11 & WDG40-3v & 1.37 & 1.37 & 1.0 & 1.786 & 0.714 & 1.0 & 1.4 & 1.64 & 2.2 & 1.45 & 0.53 & 40 \\
\hline & WDG44-3v & 1.37 & 1.37 & 1.0 & 1.786 & 0.714 & 1.0 & 1.4 & 1.64 & 2.2 & 1.45 & 0.53 & 44 \\
\hline & WDG47-3v & 1.37 & 1.37 & 1.0 & 1.786 & 0.714 & 1.0 & 1.4 & 1.64 & 2.2 & 1.45 & 0.53 & 47 \\
\hline 10 & WDG55-3v & 1.37 & 1.37 & 1.0 & 1.786 & 0.714 & 1.0 & 1.4 & 1.64 & 2.2 & 1.45 & 0.53 & 55 \\
\hline 12 & WDGN1 & 8.06 & 8.06 & 1.0 & 1.786 & 0.714 & 1.0 & 1.4 & 5.57 & 24.3 & 54 & 6.61 & 26.5 \\
\hline & WDGN2 & 4.72 & 4.72 & 1.0 & 1.786 & 0.714 & 1.0 & 1.4 & 4.9 & 9.41 & 18.4 & 3.76 & 10 \\
\hline 13 & WDGN3 & 3.47 & 3.47 & 1.0 & 1.786 & 0.714 & 1.0 & 1.4 & 4.42 & 6.54 & 11.54 & 2.89 & 30 \\
\hline
\end{tabular}


To simulate a rigid wedge we take a logically rectangular mesh and bend the left logical side through the desired angle, $\theta_{w}$, away from the left vertical axis. Then the interior coordinates of the mesh are rezoned to conform to the boundary configuration. Figure 2 illustrates the transformation of the logically rectangular mesh to the computational mesh.

All of the results in this section were obtained employing a purely Eulerian mesh, second-order Godunov technique and advection with van Leer 11miting.

The experfmental data obtalned by Hellig and Relchenbach have been plotted In terms of the quantity $\Delta \mathrm{P}_{r} / \Delta \mathrm{P}_{\text {Inc }}$ as a function of incident angle, $\theta_{I}$ (and $\theta_{w}$ ), where $\Delta \mathrm{P}_{\text {Inc }}$ is the change in pressure from the pre-shocked region to that of the post-shocked region; 1.e., $\Delta \mathrm{P}_{\text {Inc }}=\mathrm{P}_{-}-\mathrm{P}_{0} \cdot \Delta \mathrm{P}_{\text {Inc }}$ is the same for all wedge angles at any particular Incident-shock Mach number, $M . \Delta \mathrm{P}_{r}$ is the change in * surface pressure from the pre-shocked region to the reflected-shock regton and varles depending on the wedge angle and shock structure. Figure 3 shows how $\Delta P_{r}$ and $\Delta \mathrm{P}_{\text {Inc }}$ are calculated depending on the shock structure. In the experiments described by these authors, four different Mach numbers were used for the Inctdent shock. We have chosen three of these for our comparisons, 3.36, 1.26, and 1.37.

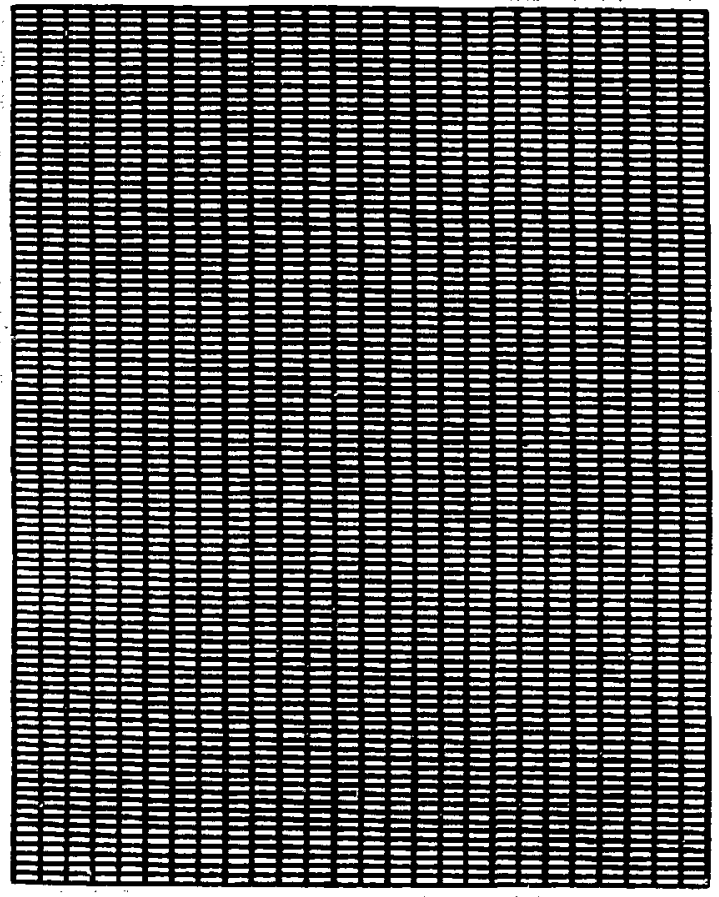

FIg. 2(a)

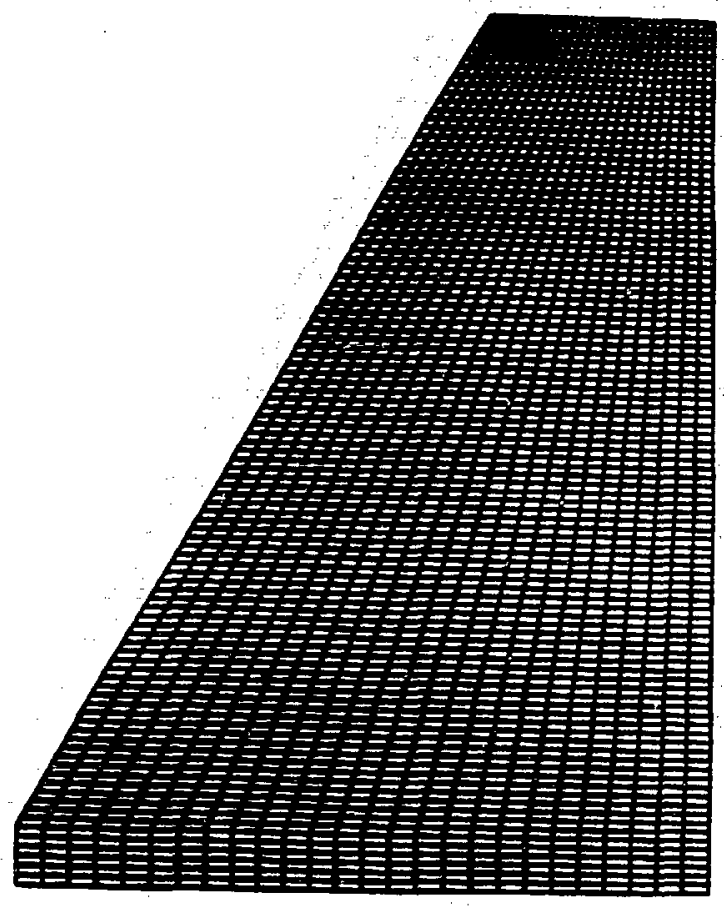

Fig. 2(b)

F1g. 2. Logically rectangular mesh (a) transformed to a calculational mesh and (b) used in the wedge studies. 


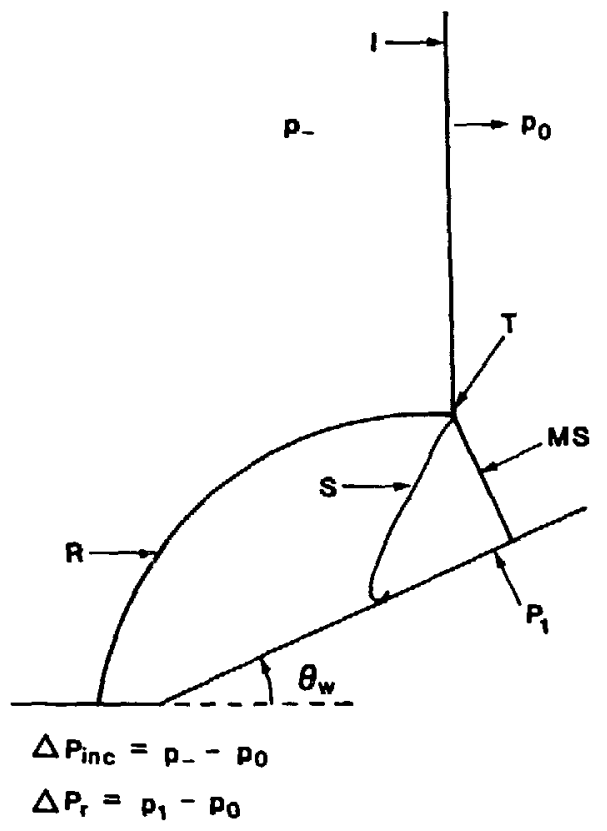

Fig. 3(a)

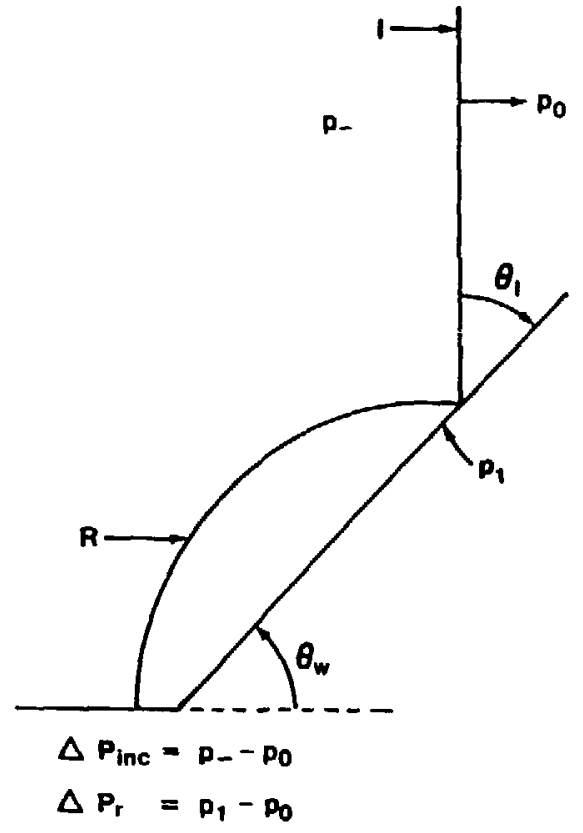

Fig. 3(b)

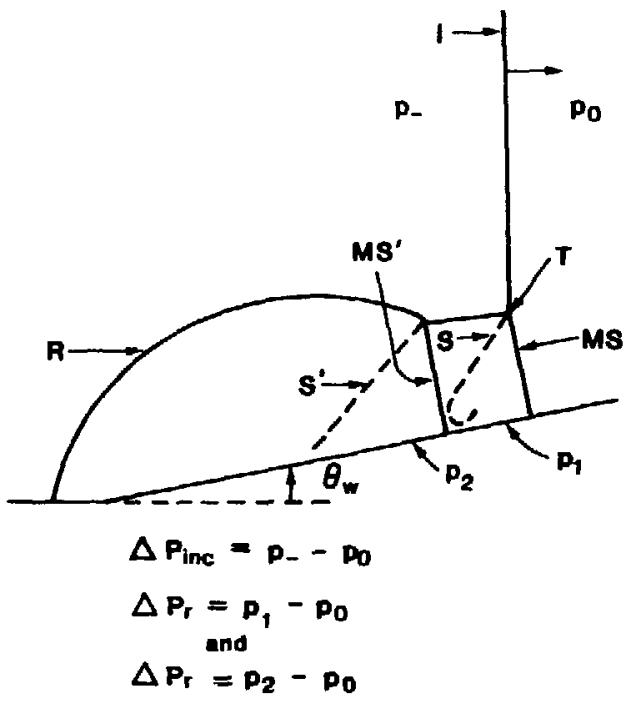

F'ig. 3(c)

F1g. 3. Shock configuration with formulas for $\Delta P_{r}$ and $\Delta P_{1 n c^{*}}$ (a) regular reflection, (b) simple Mach reflection, and (c) double Mach reflection. I 1s Incident shock, R 18 reflected shock, MS is primary Mach shock, T is triple point, S is primary slipline, MS' is secondary Mach shock, and $S$ ' is secondary slipline. 
The first of these shows double Mach reflection, with good agreement among experImental, theoretical, and calculational results. The second showed some interestIng discrepancles that are discussed in Sec. VI. Because of those discrepancles we examined the third case, with Mach number close to that of the second, and saw considerably less discrepancy.

Figure 4 shows the comparison of our computational data to the two- and three-shock theory and to the experimental data presented by Hellig and Relchenbach for the first of the three different Mach numbers. The solid lines are theoretical curves; the solid line on the left is from two-shock theory (regular reflection) and the solid line on the right is from the three-shock theory (Mach reflection). The circles with error bars are experimental data that Hellig and Relchenbach present, and the + points are our computational results. At this high incident-shock Mach number, $M=3.36$, the three-shock theory does not predict the double Mach reflection that occurs at the shallow wedge angles, however, it does predict the pressure jump across the primary Mach stem. The experimental data agree well with the two-shock theory at incidence angles less than 40 degrees and with the three-shock theory across the primary Mach stem.

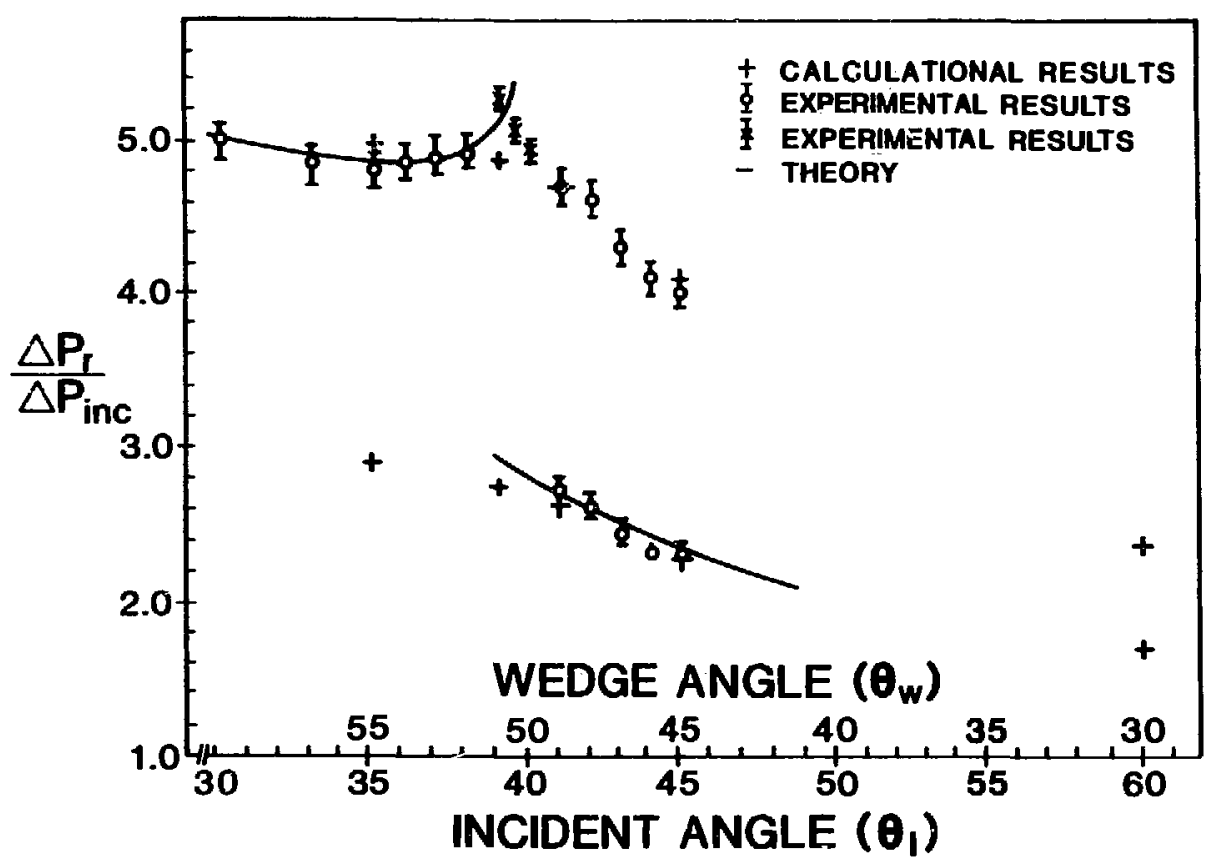

F1g. 4. Comparison of $\Delta P_{r} / \Delta P_{\text {Inc }}$ as a function of incident angle. The incident shock Mach number 1s 3.36 . 
A secondary Mach shock is present at Incident angles greater than 40 degrees as Indicated by the experimental results. Our computational data agree well with both the two- and three-shock theory as well as with the experimental data. The calculations Indicate that the double Mach structure occurs at $\theta_{I}=35^{\circ}$ (see F1g. 5), whereas the experimental data do not Indicate this. At the angle of transition from regular to double Mach reflection $\left(\theta_{w} \approx 51^{\circ}\right)$ our computational results are lower than theory predicts. These discrepancles are discussed in Sec. VI.

F1gure 6 shows a qualitative compsrison of a sompucet-generatec depiction of shock structure to a Schlieren photograph [2] of an actual experiment. Both are at the same wedge angle $\left(\theta_{\mathrm{w}}=45^{\circ}\right)$. The 1ncident-shock Mach number in our CAVEAT calculation is 3.36, whereas the Incident-shock Mach number of the experiment is 3.03. Despite this difference, the two shock structures are very similar. We can easily locate the two triple points and the sliplines that emerge from the triple points due to the change in entropy in that region.

Figure 7 shows a comparison of our computational resulta with the experimental data and two-shock theory for the second of the three Mach numbers.

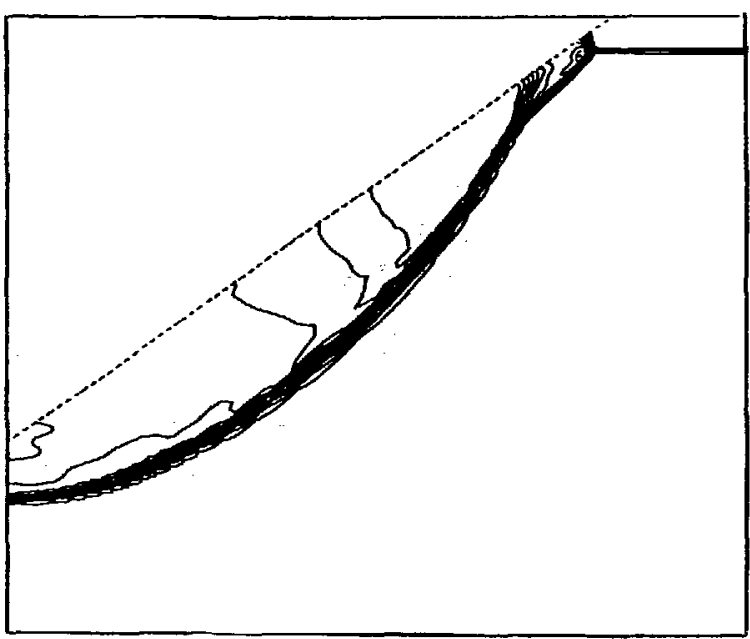

$\mathrm{F} 1 \mathrm{~g} \cdot 5(\mathrm{a})$

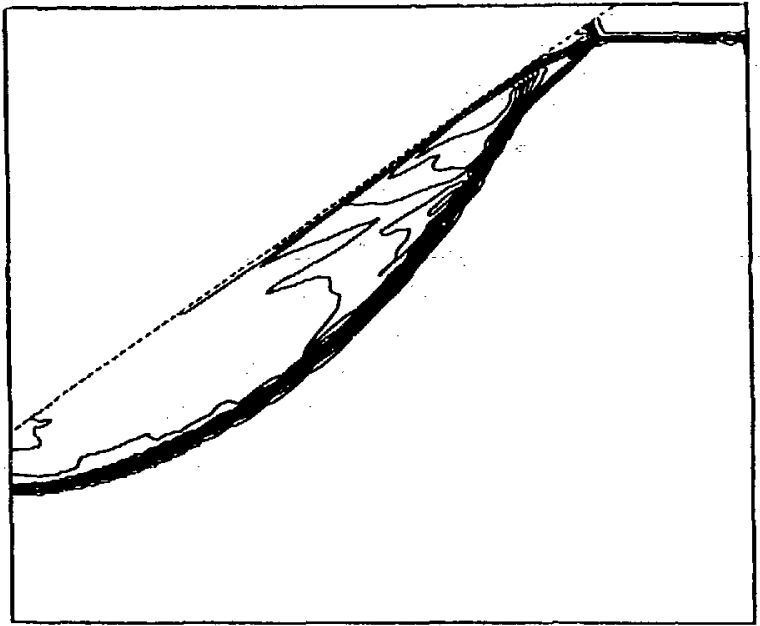

F1g. 5(b)

FIg. 5. Computer-generated depiction from a calculation where $M=3.36$ and $\theta_{w}=55^{\circ}$; (a) Is pressure contour and (b) density. Note that double Mach reflection occurs. 


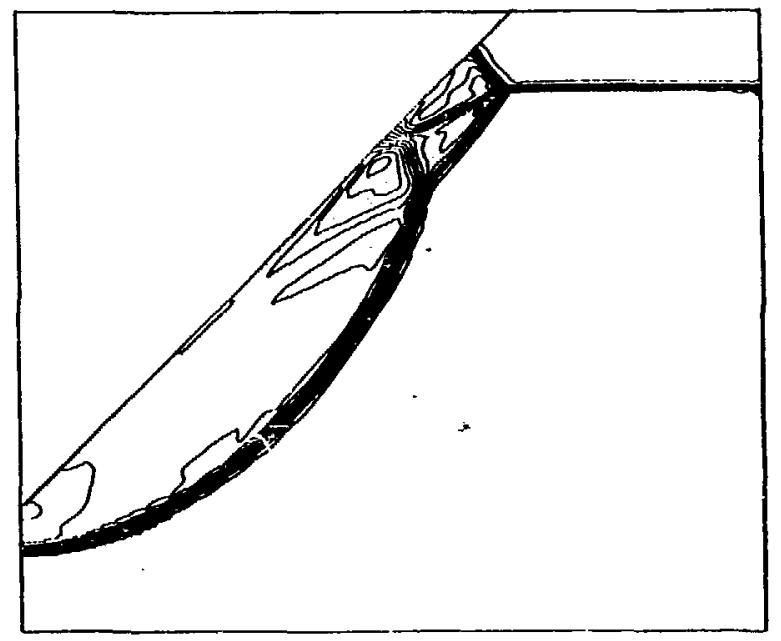

Fig. $6(a)$

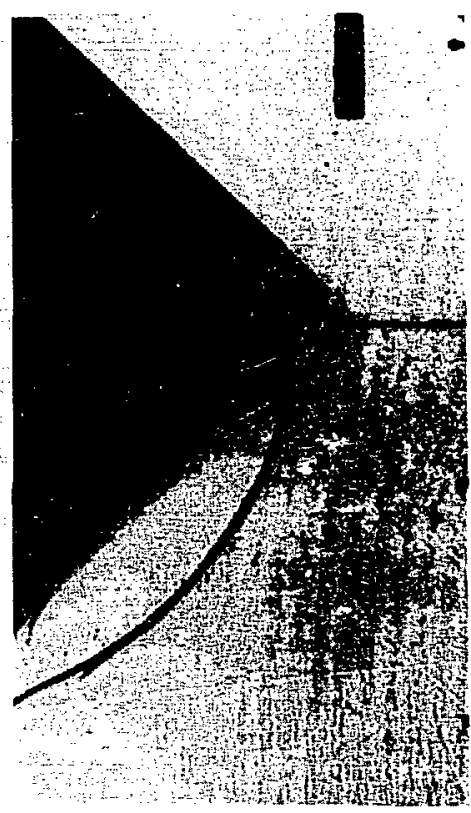

Fig. $6(\mathrm{~b})$

F1g. 6. Comparison of shock structure between calculation and experiment.

(a) computer-generated density contours for $M=3.36$ cas s and

(b) Shadow-Schlleren photograph for $M=3.03$ case. [2]

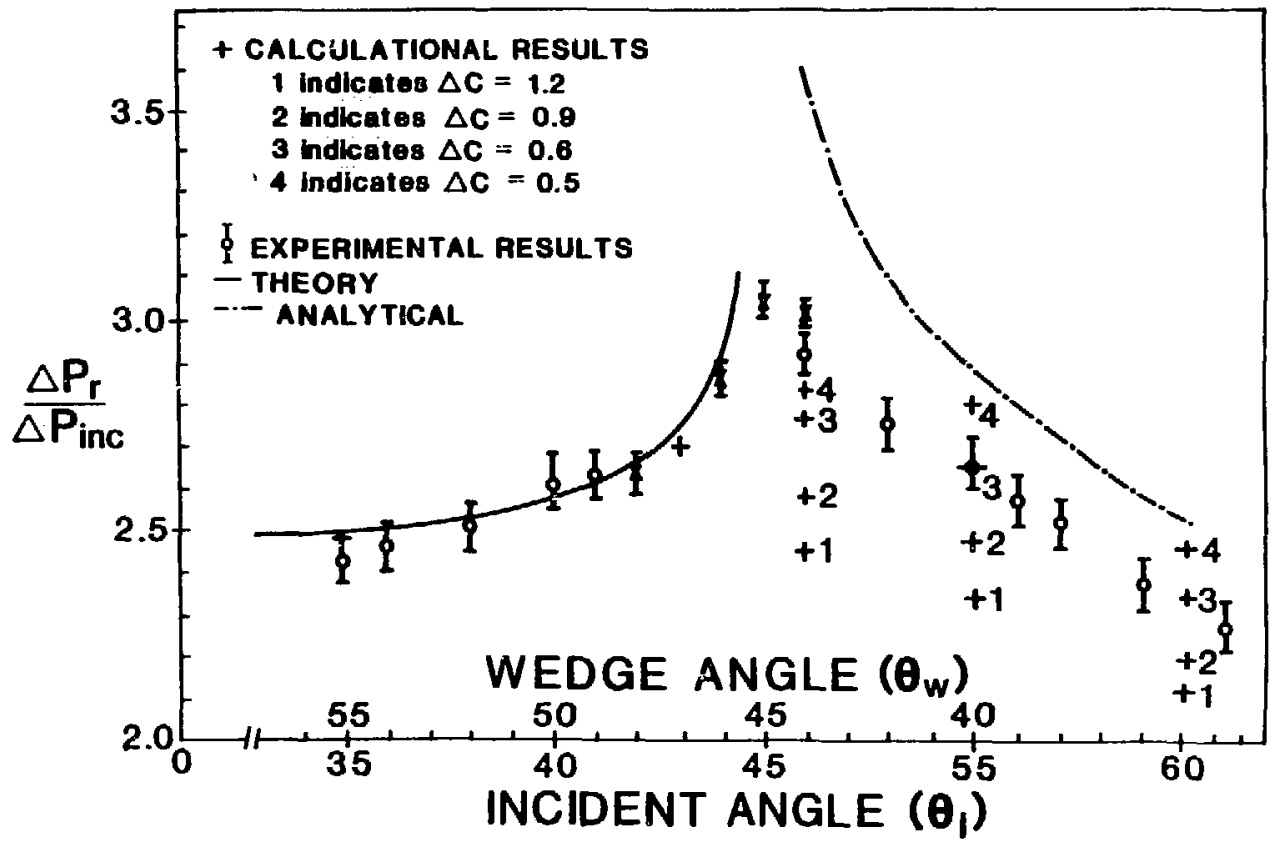

Fig. 7. $\Delta \mathrm{P}_{\mathrm{r}} i \Delta \mathrm{P}_{\text {Inc }}$ as a function of Incident angle. The Incident Mach number is $M=1.26$. 
At this smaller Incident-shock Mach number of 1.26 , the formation of the double Mach reflection does not occur, Instead simple Mach reflection occurs. Agaln, the two-shock theory, the experimental data, and our computational re iits agree we11. The dashed 11 ne presented on this figure at incident angles $g:$ " "r than 45 degrees was obtalned by using computer-generated pressure contour pilots from our computational data and measuring the distance the Mach stem is from a fixed point at various times. Graphing distance versus time we find $V_{m s}$ from the slope. Knowing $V_{\mathrm{ms}}$, we can use Eqs. (1) and (4) to calculate the pressure jump across the Mach stem thus allowing us to derive an analytical interpretation in this region. A comparison of the raw computational result8, the experimental data, and the analytical interpretation shows that our computational results and the experimental data tend to be 1ow. Near the critical angle of 46 degrees the low values occur for the same reason as in Fig. 4; for larger angles the discrepancy results from the extreme narrowness of the pressure pulse; both of these effects are discussed in Sec. VI.

Figure 8 shows the shock structure and the exceptional resolution that can be obtained using computer-generated pressure contours obtained from the CAVEAT salcu?ations at three different wedge angles. The initial conditions are the same for all three calculations. At the smaller wedge angle we see that a large Mach stem occurs and as the angle increases, the simple Mach reflection structure becomes smaller until only regular reflection occurs. Note that at this low Mach number, double Mach reflection does not occur

As explained above, we examine a third Macil number close in value to the second. Figure 9 shows a comparison of our computational results to experimental data and to theoretical and analytical curves. Again at this Mach number of 1.37, only transition from regular reflection to simple Mach reflection occurs. In contrast to the results shown in $\mathrm{FIg} .7$, our computational results are here in good agreement with the experimental data and the analytical curve except at the angles of transition from regular to Mach reflection $\left(\theta_{I} \approx 43^{\circ}\right)$, where our computational results are lower than theory predicts. Figure 10 shows computergenerated deplction of the regular reflection at an angle of incidence of 35 degrees. The reflected shock appears thicker towards the bottom of the wedge because the computational cells are larger in that region. Figure 11 shows a computer-generated depiction of a simple Mach reflection at an angle of incidence of 50 degrees. 


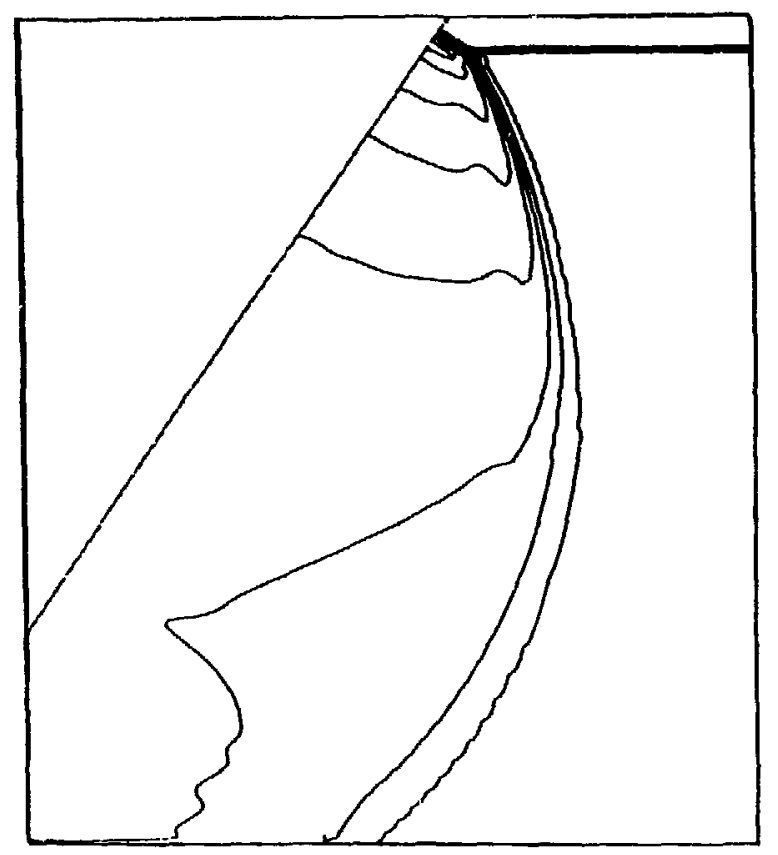

FIg. 8(a)

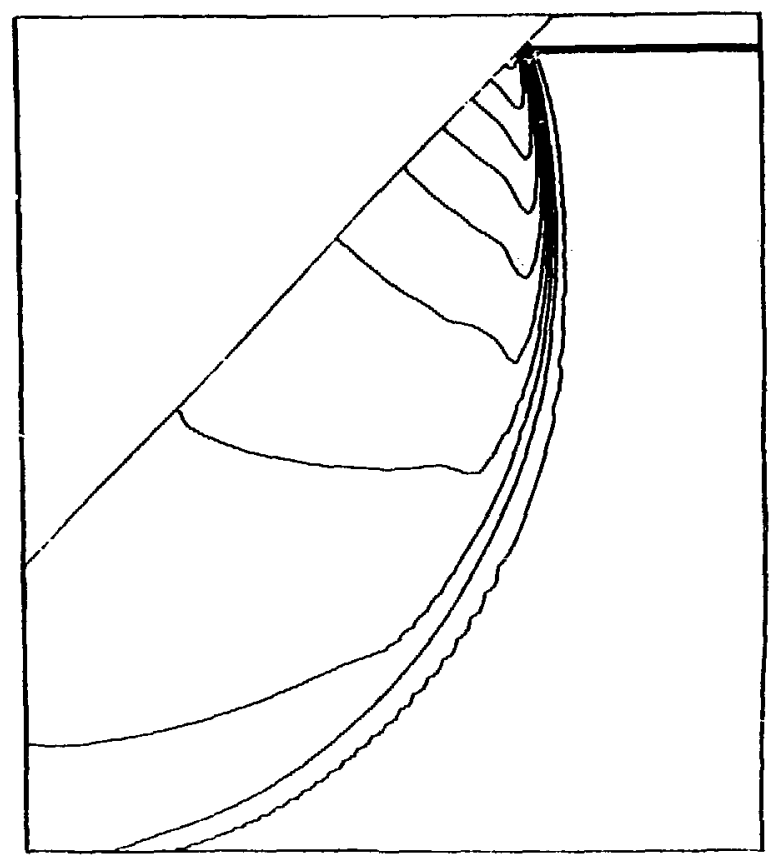

Fig. 8(b)

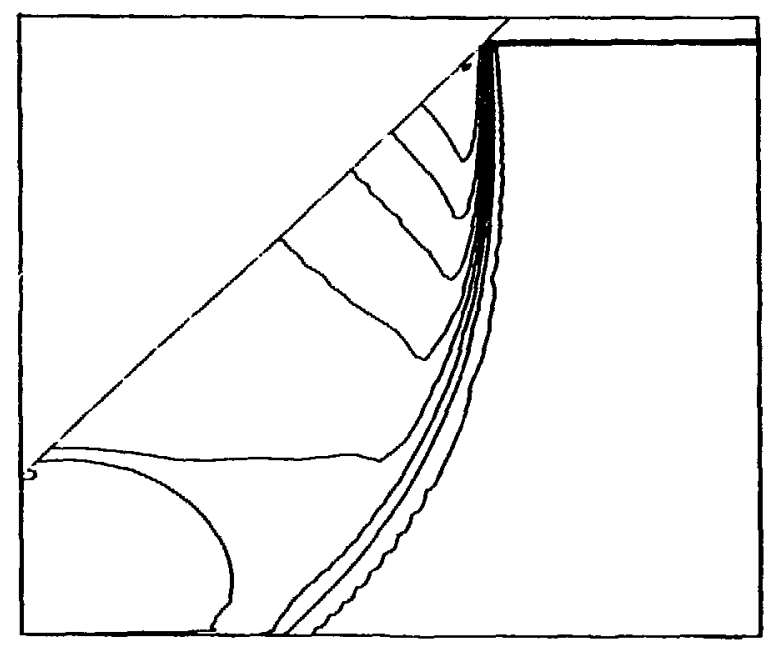

Fig. 8(c)

F1g. 8. Computer-generated pressure contours depicting shock structure for $M=1.26$. (a) $\theta_{w}=35^{\circ}$, (b) $\theta_{w}=44^{\circ}$, and (c) $\theta_{w}=47^{\circ}$. 


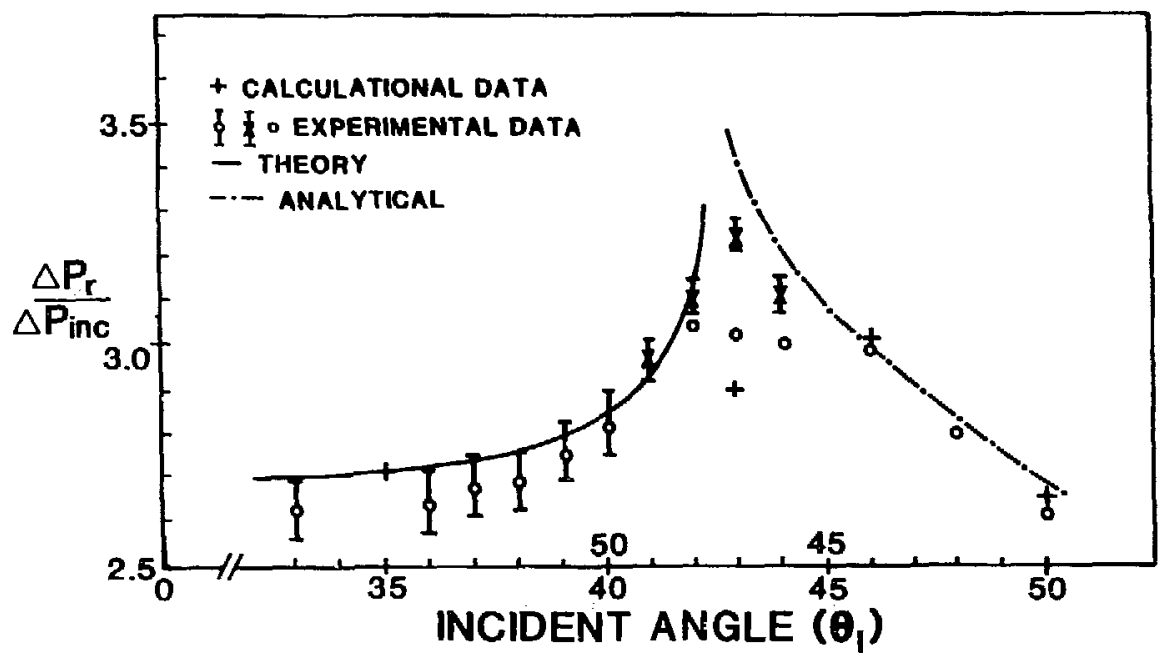

Fig. 9. $\Delta P_{r} / \Delta P_{\text {inc }}$ as a function of incident angle. The incident Mach number is 1.37 .
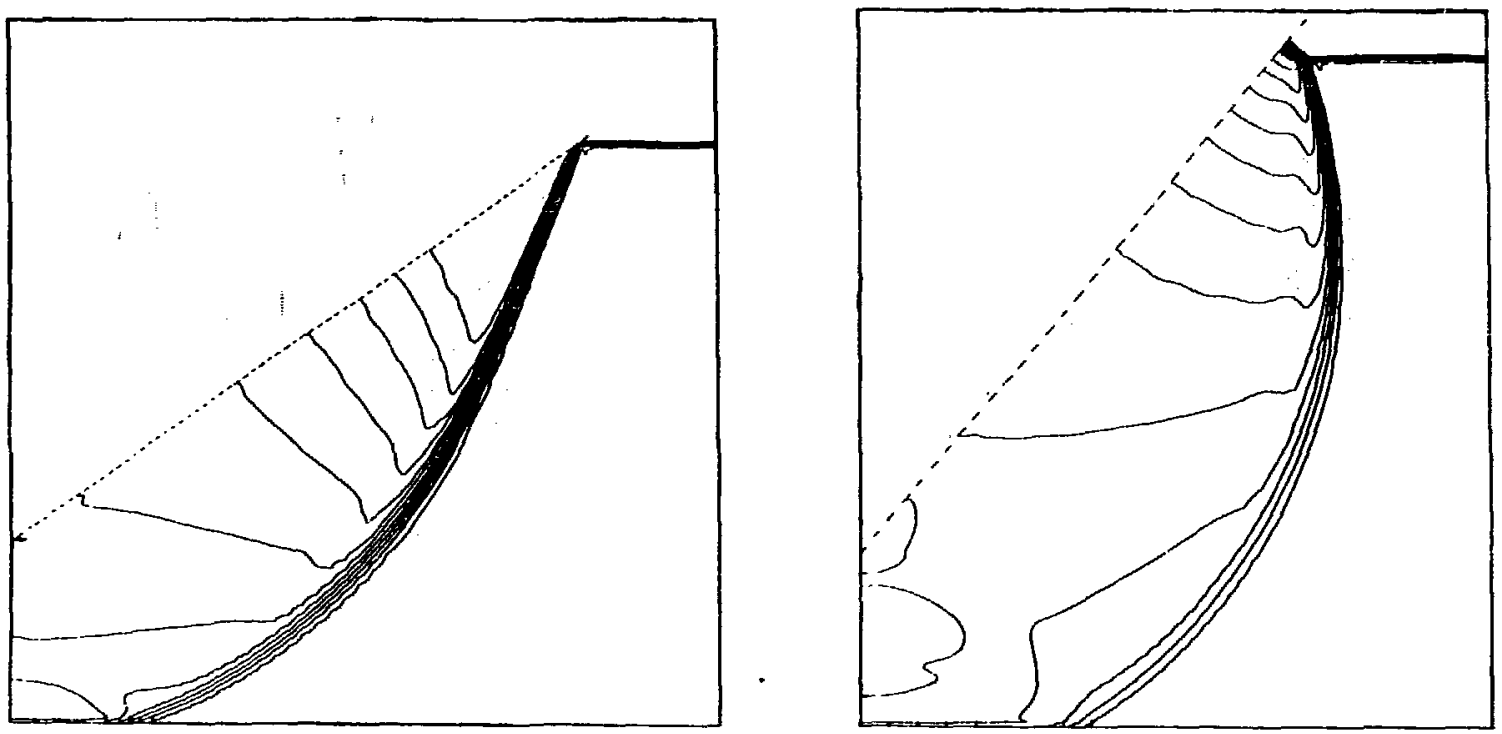

Fig. 10. Pressure contours for regular reflection. $\theta_{w}=55^{\circ}$ and $M=1.37$.
Fig. 11. Pressure contours for simple Mach reflection. $\theta_{w}=40^{\circ}$ and $M=1.37$. 
Using computer-generated density contour plots, we qualitatively compare shock structures produced by our CAVEAT calculations to those shown on Interferograms of actual experiments. We make comparlsons for two different cases; the first case we Investigate has a wedge angle of 26.5 degrees and an Incoming Incident shock with a Mach number of 8.06. Figure 12 1s the comparison of shock structures between our calculation and the experiment of Ben-Dor and Glass [3]. This comparison shows that CAVEAT Is producing very simllar features to those of the interferogram, in particular, the s1ipline emerging from the triple point. The Interferogram shows that the slipline curves in towards the Mach stem in a manner that is essentially identical to that of the calculation. The Interferogram also shows a shock structure extending from the slipline to the first

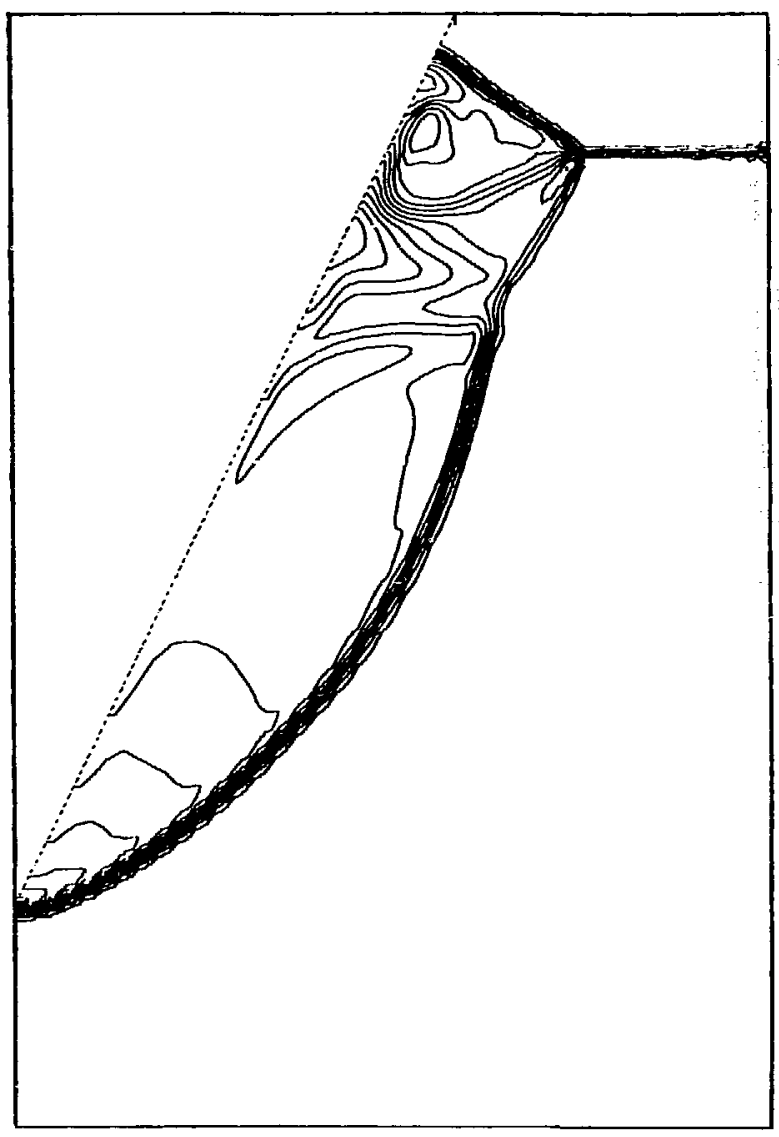

F1g. $12(a)$

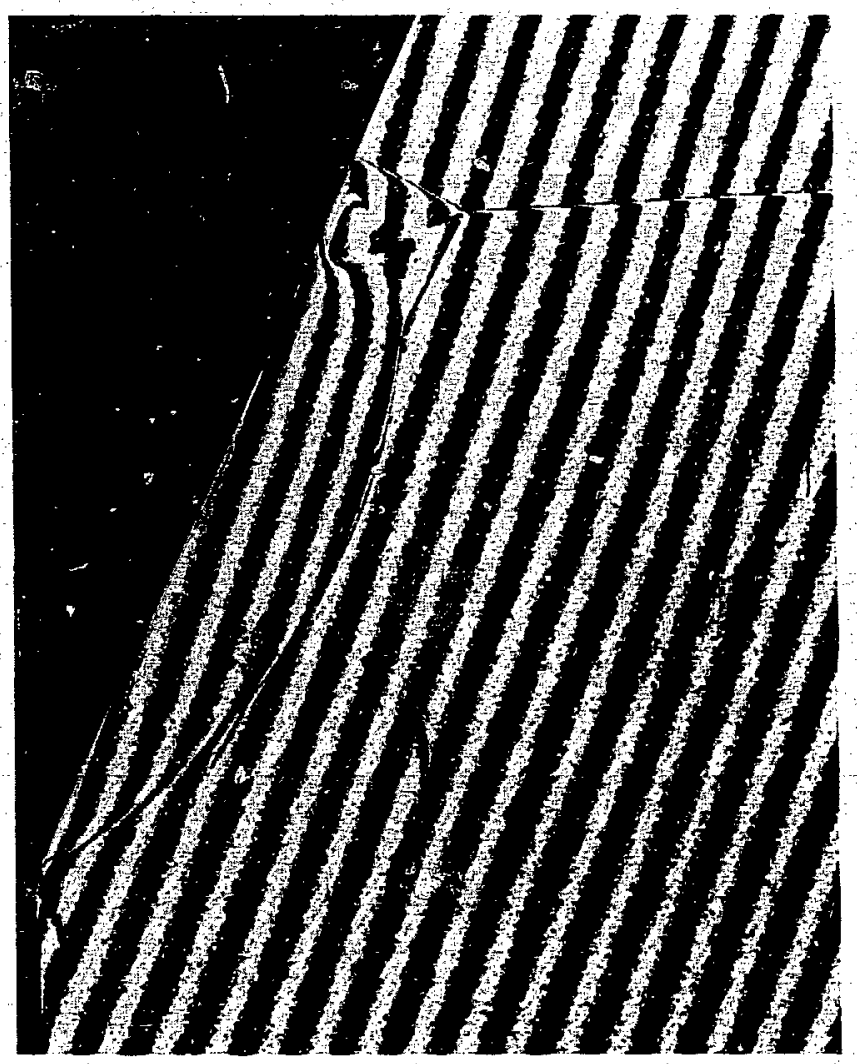

F1g. $12(\mathrm{~b})$

F1g. 12. Comparison of shock structures. (a) computer-generated density contours and (b) interferogram [3] of an actual experiment. $M=8.06$ and $\theta_{w}=26.5^{\circ}$. Figure 12(b) copyright 1979, Cambridge University Press. Reprinted with permission. 
reflected shock, deflecting it to an outward curving arc. Our computational results also show this shock structure. For the second comparison with these authors we have a wedge angle of 30 degrees and an Incoming incident-shock Mach number of 3.47. Figure 13 is a comparison of shock structure between our calculation and the experiment. This comparison also shows similar detall of shock structure. Our calculation shows a strong slipline with a sharp curvature along the wedge towards the Mach stem. The Interferogram also shows that the slipline has a small radius of curvature where it bends towards the Mach shock, however, the Interferogram does not show the strong density gradient at the surface. This difference may be explained by the fact that the Interferogram IInes In Fig. 13 are nearly perpendicular to the wedge, in contrast to the nearly parallel lines In Fig. 12.

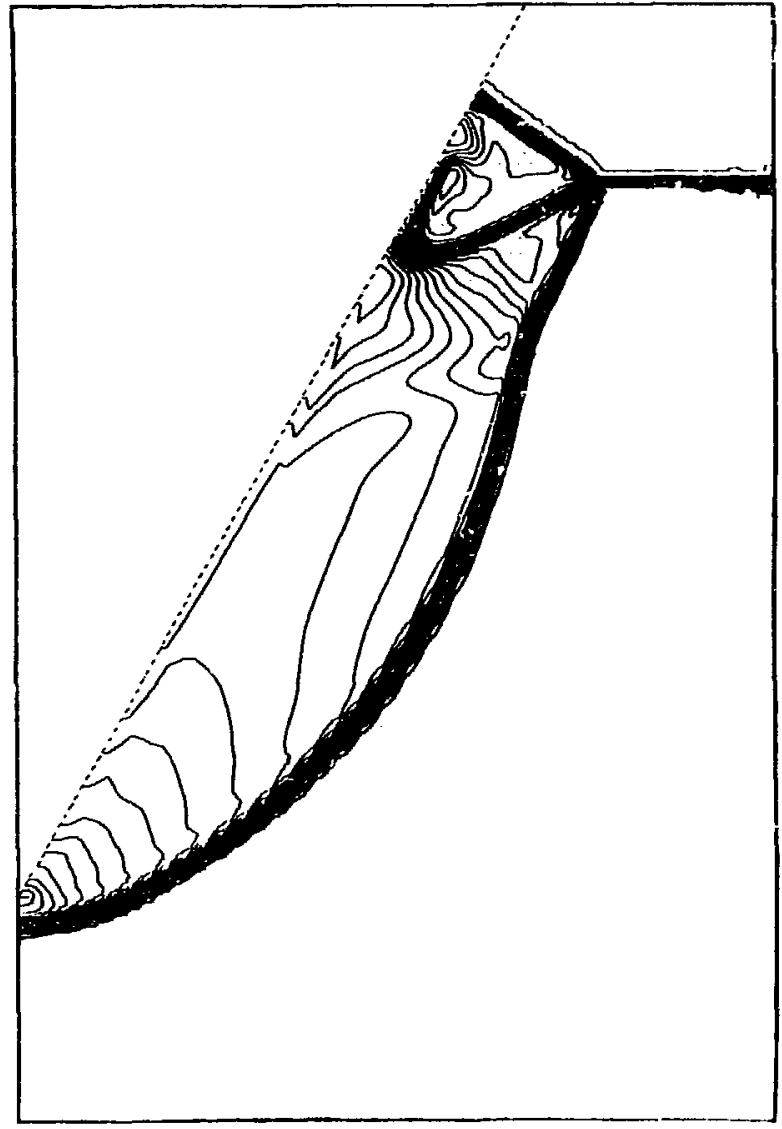

F1g. $13(a)$

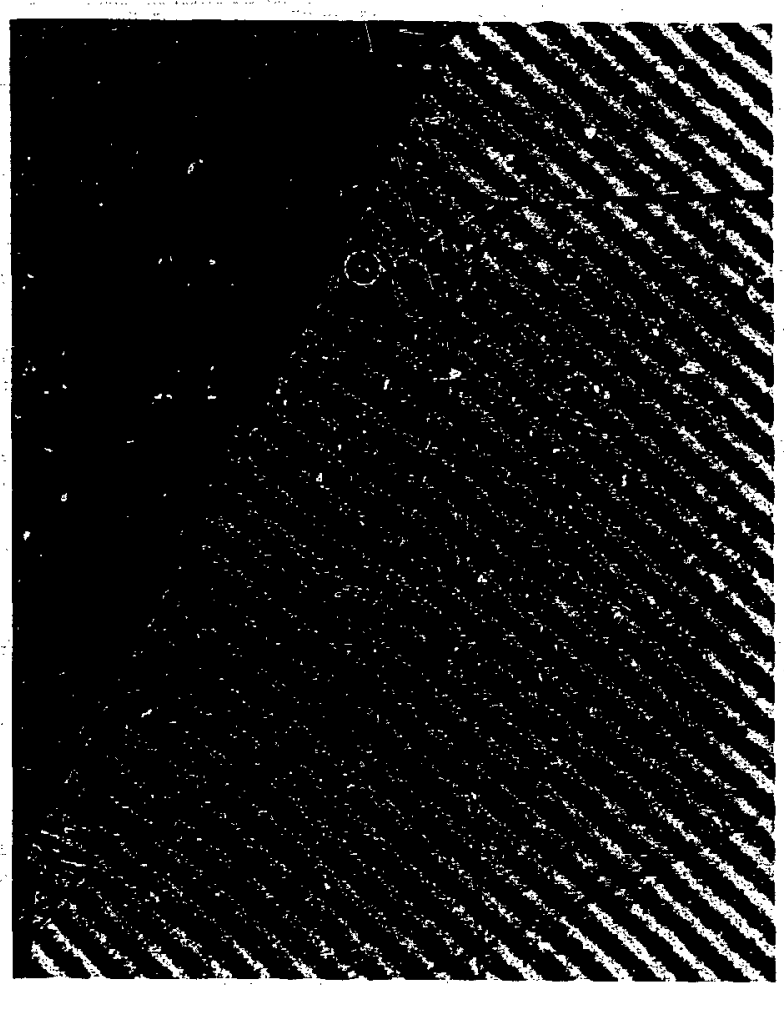

Fig. $13(b)$

F1g. 13. Comparison of shock structures. (a) computer-generated density contours and (b) Interferogram [3] of an actual experiment. $M=3.47$ and $\theta_{w}=30^{\circ}$. Figure 13(b) copyright 1979, Cambridge University Press. Reprinted with permission. 
These results on the rigid wedges give us confidence that the CAVEAT code has the capability to produce an accurate description of the complex interaction process of shocks in gases interacting with rigid wedges. By comparing our CAVEAT computational result to the experimental data presented by Hellig and Relchenbach we find considerable agreement. Then by comparing our computergenerated density contour deplctions to Schlieren photographs and Interferograms, we find that CAVEAT produces simflar shock structures to those of the experiments.

\section{RIGID CYLINDERS AND SPHERES}

For the rigid cylinder and sphere studies we are concerned with the changing shock structures and pressures generated by the shock Interaction with these curved surfaces resulting from the continuously changing angle of intersection, for two Incoming shock Mach numbers, $M=2.37$ and $M=3.11$. We compare the cyIIndrical computations with experimental data from Hellig and Reichenbach [2] and compare and discuss the spherical computations with our cylindrical results.

Keeping our pre-shocked conditions the same as in our wedge calculations, we can use Eqs. (1) through (4) to calculate our Incoming shock conditions. Table III lists all the input conditions used In our rigid cylinder and sphere studies. Figure 14 shows our set-up configurations for these studies, where the bottom boundary is Inflow and the top boundary is outflow with the right boundary reflective and left boundary elther reflective or a cylindrical axis.

TABLE III

INPUT DATA FOR RIGID CYLINDER AND SPHERE CALCULATIONS

\begin{tabular}{|c|c|c|c|c|c|c|c|c|c|c|c|c|}
\hline $\begin{array}{c}\text { Figure } \\
\text { Number }\end{array}$ & $\begin{array}{c}\text { Run } \\
\text { Number }\end{array}$ & $M$ & $v_{S}$ & $\rho_{0}$ & $I_{0}$ & $p_{0}$ & $c_{0}$ & $\mathrm{~V}$ & $\rho_{-}$ & $I_{-}$ & $p_{-}$ & $U_{-}$ \\
\hline 14,15 & CYCS4M & 2.37 & 2.37 & 1.0 & 1.786 & 0.714 & 1.0 & 1.4 & 3.17 & 3.59 & 4.56 & 1.62 \\
\hline 16 & CYCS4M & 2.37 & 2.37 & 1.0 & 1.786 & 0.714 & 1.0 & 1.4 & 3.17 & 3.59 & 4.56 & 1.62 \\
\hline & CYCS5 & 3.11 & 3.11 & 1.0 & 1.786 & 0.714 & 1.0 & 1.4 & 3.96 & 5.02 & 7.94 & 2.32 \\
\hline & CYCS5M & 3.11 & 3.11 & 1.0 & 1.786 & 0.714 & 1.0 & 1.4 & 3.96 & 5.02 & 7.94 & 2.32 \\
\hline $16,18,20,21$ & CYCS5N & 3.11 & 3.11 & 1.0 & 1.786 & 0.714 & 1.0 & 1.4 & 3.96 & 5.02 & 7.94 & 2.32 \\
\hline 23 & SPHS6 & 3.11 & 3.11 & 1.0 & 1.786 & 0.714 & 1.0 & 1.4 & 3.96 & 5.02 & 7.94 & 2.32 \\
\hline
\end{tabular}




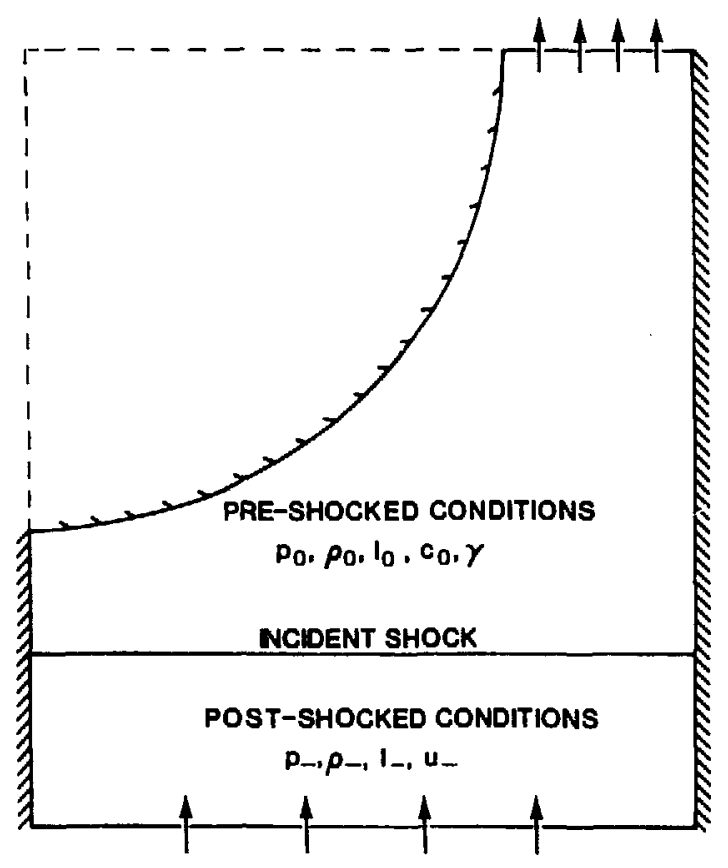

Fig. 14. Configuration for the rigid cylindrical and spherical problems. The top boundary is outflow, and the bottom inflow. The right and curved boundaries are reflective, while the left boundary may be reflective or a cylindrical axis.

The rigld cylinder and ephere problems were run with an Eulerian mesh, with second-order Godunov technique and with second-order advection. The cylinder problems were run in plane geometry and the spherical problems were run in cyilndrical geometry in which the left axis is a cylindrical axis of symmetry.

The Incident Mach number is 2.37 for our first cylinder calculation. Figure 15 shows that our mesh set-up consists of a logically rectangular mesh with only one region and one part to that reglon. We produce this mesh by bending part of the top boundary of the logically rectangular mesh into a quarter circle beginning and ending at the desired radial distance from the top left corner.

We first ran this problem using van Leer limiting; however, this caused some difficulty in reproducing the surface pressure jump across the Mach shock, because of an overshoot in the pressure. Switching to a monotone limiting scheme helped smooth out the overshoot, thus enabling us to obtain the correct peak

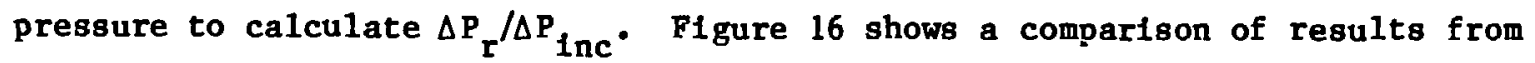
the monotone and the van Leer limiting schemes, in the form of pressure versus $\theta_{I}$ at a given instant in time. 


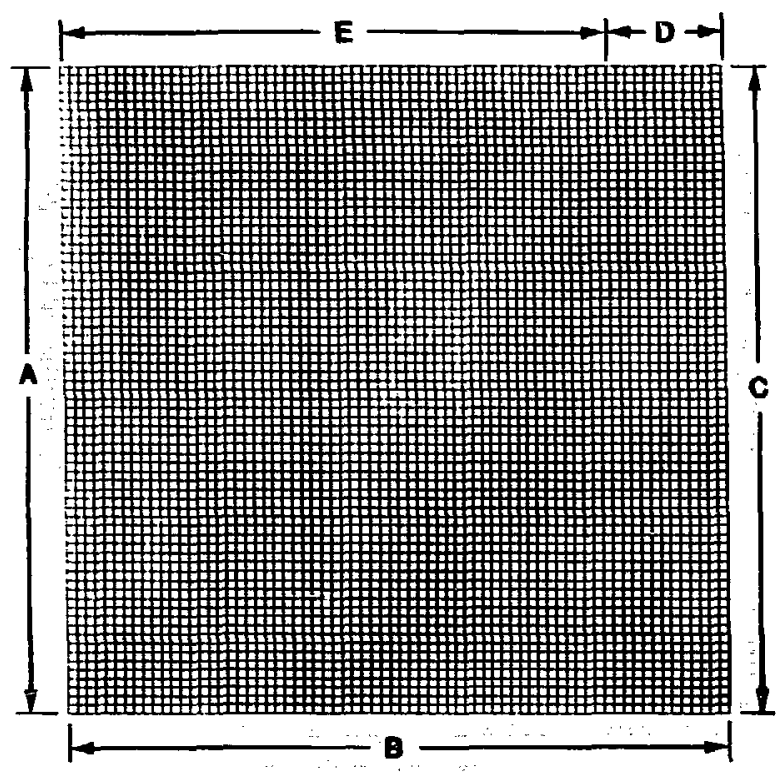

Fig. 15(a)

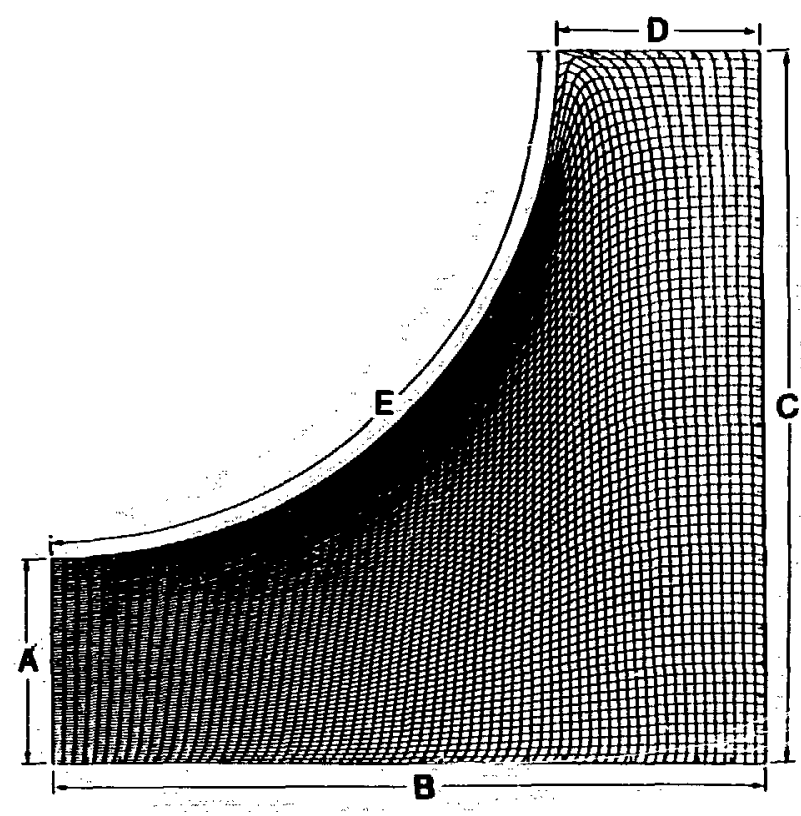

F1g. 15(b)

Fig. 15. (a) logically rectangular mesh and (b) calculational mesh used in first rigid cylindrical calculation. Corresponding letters denote the common sides are labeled.

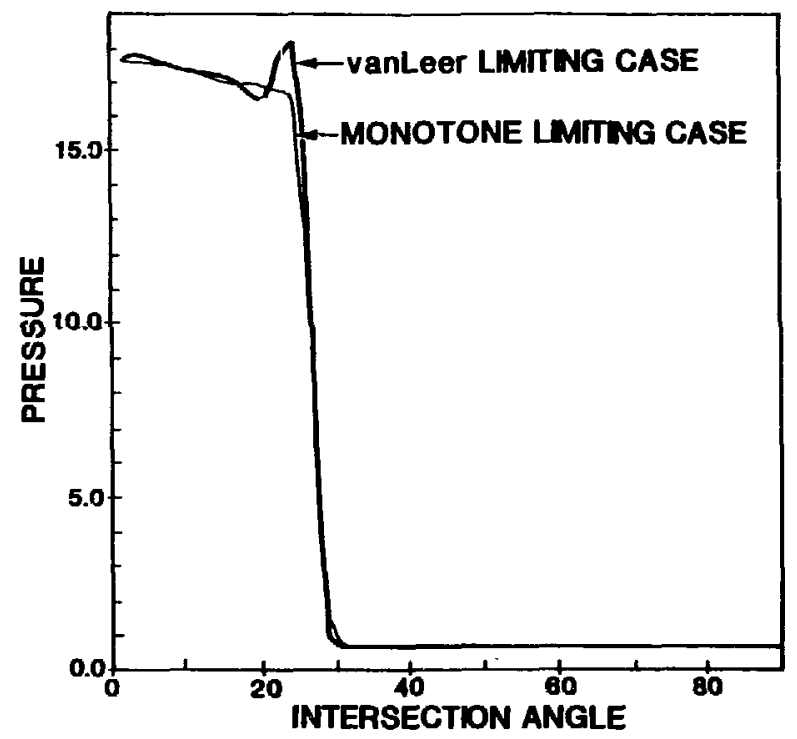

Fig. 16. Pressure vs angle of intersection, showing the difference between van Leer and monotone limting. 
Figure 17 is a comparison of our CAVEAT computational results with two-shock theory and experimental data. The experimental data presented by Heilig and Relchenbach were obtained by measuring the angle of the reflected shock during the regular reflection phase and applying a form of Snell's law to these reaults; these points are the circles in the figure. Our computational resuits are the points with the + signs, Here we present results from toc cypes of calculations, one with monotone and the other with van Leer limfting. The + points are results obtalned using the monotone limiting al: sine dot points were obtained using van Leer 1imiting, extrapolates to omlt the overshoot. At angles of incidence in which we ised the van weer limiting calculations to determine $\Delta P_{r} / \Delta P_{\text {inc }}$ we did not have a large overshoot in pressure across these shocks and the peak pressure was rot difficult to estimate.

Our calculational results indicate that as the angle of Intersection increases the peak surface pressure decreases. Up to an angle of approxfmately 38 degrees our calculational results 1 le on the theoretical curve; for larger angles the calculational points depart smoothly from that curve and in particular do not agree with the sharp rise indicated by the theory at about 40 degrees. The experimental data presented by Hellig and Relchenbach are lower than the two-shock theory and our CAVEAT results, as discussed in Sec. VI.

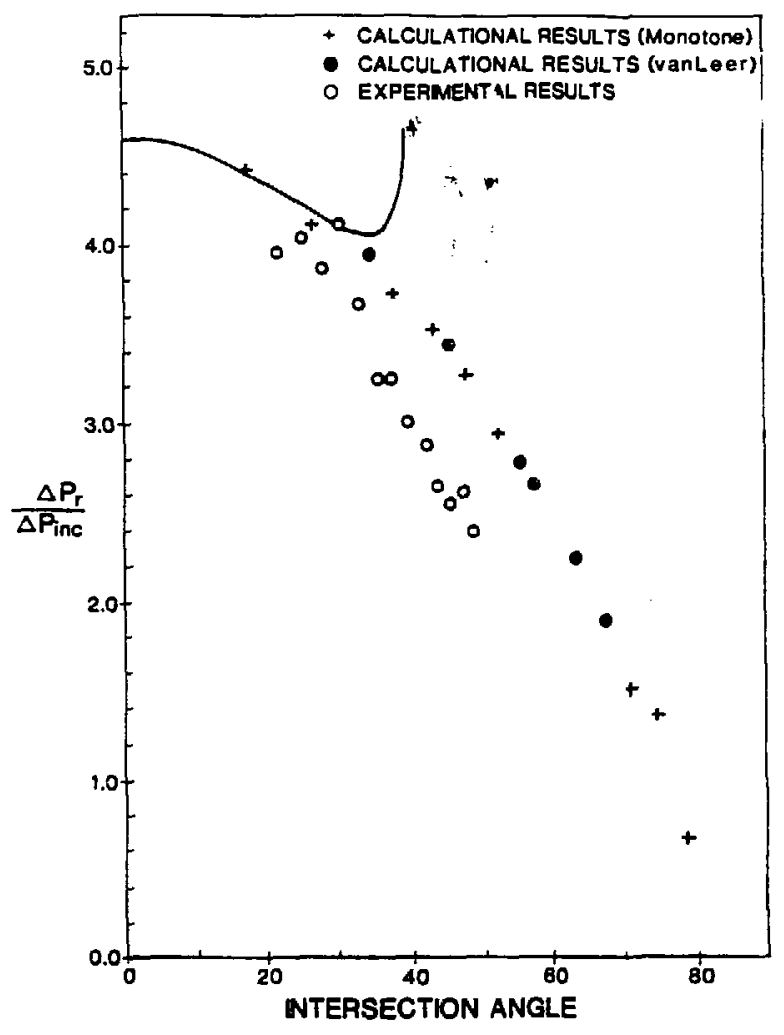

Fig- 17. $\Delta P_{r} / \Delta P_{\text {Inc }}$ as a function of Intersection angle for an incldent shock ( $M=2.37$ ) passing over a rigid cylinder. 
Our second cylinder CAVEAT calculation uses new Incldent-shock conditions with $M=3.11$. In this case we employ only van Leer limiting. To demonstrate the capability for alternative meshing In CAVEAT, we use a more uniform and orthogonal mesh around the cylinder. This required making our logically rectangular mesh Into two separate reglons with one part each. The top boundary of each region represents the curved rigid cylinder boundary. The bottom boundary of region one is our prescribed Inflow boundary shown In FIg. 14. The right boundary of region two is our prescribed top outflow boundary also shown In Fig. 14. The right boundary of region one and the left boundary of region two are communtcative boundarles allowing fluxing between the two reglons. The rest of the boundarles are reflective. Figure 18 shows how the logically rectangular mesh is transformed into our computational mesh.

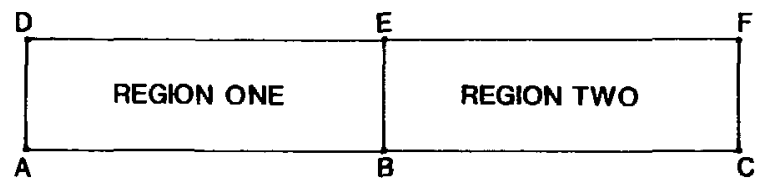

Fig. $18(a)$

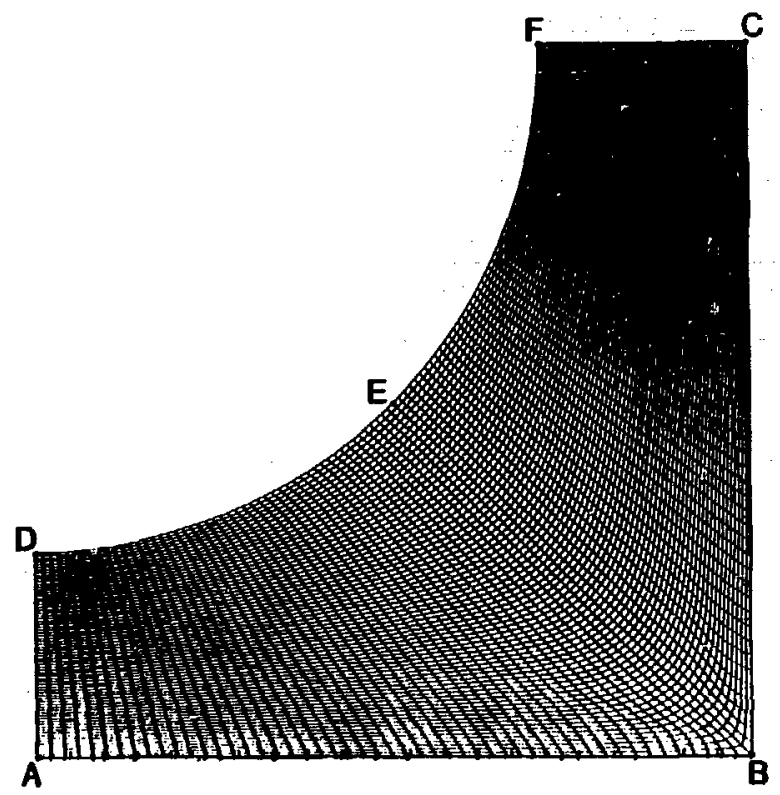

Fig. $18(b)$

Fig. 18. (a) logically rectangular mesh and (b) computational mesh used in the second cylinder and spherical problems. 
Figure 19 is the comparison of this second rigid cylinder CAVEAT calculation to two-shock theory and to experimental data. Up to an intersection angle of approximately 30 degrees our calculational results lie on the theoretical curve; for larger angles the calculational points depart smoothly from that curve and again do not agree with the sharp rise indicated by theory at about 38 degrees. At angles of intersection greater than 60 degrees, we see a second jump in pressure along the cylinder gurface (see FIg. 21). This phenomenon 1s similar to the double Mach reflection results discussed in the first wedge problem. The plotted points were obtalned by using the average of the maximum and minimum values of pressure and the uncertainty bars include the entire range from the minimum to maximum pressures in this region. Figure 20 shows an example of this overshoot.

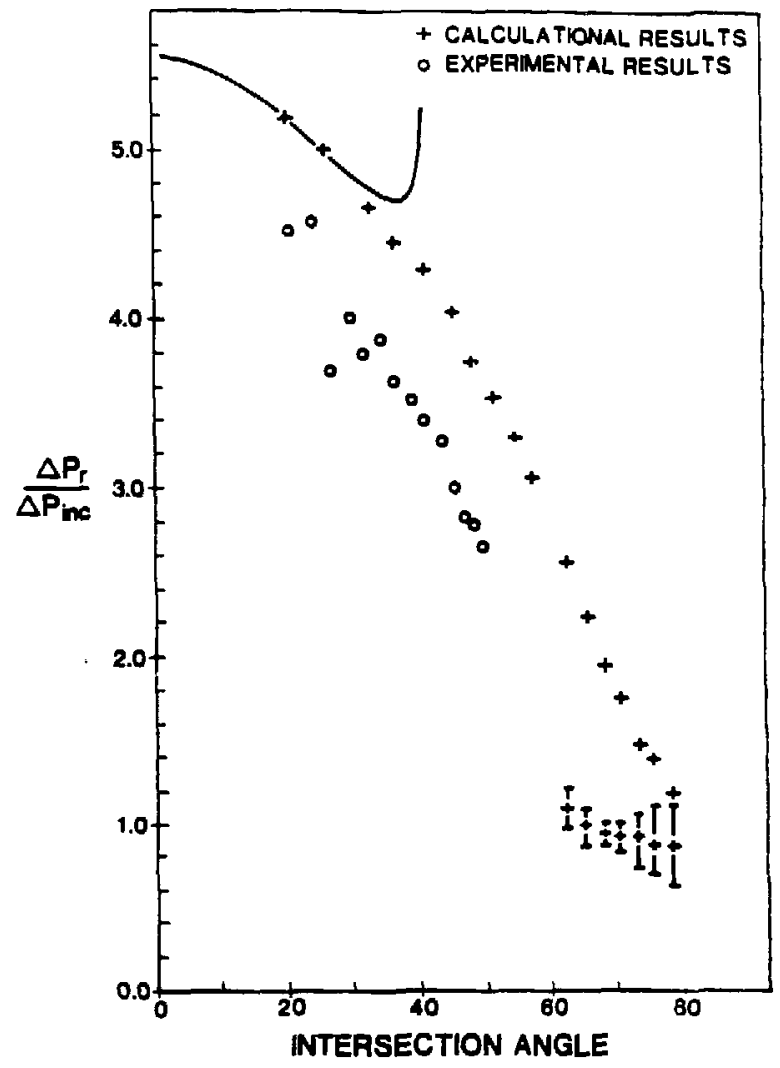

Fig. 19. $\Delta \mathrm{P}_{\mathbf{r}} / \Delta \mathrm{P}_{\text {Inc }}$ as a function of Intersection angle for an Incident shock $(M=3.11)$ passing over a rigid cylinder.

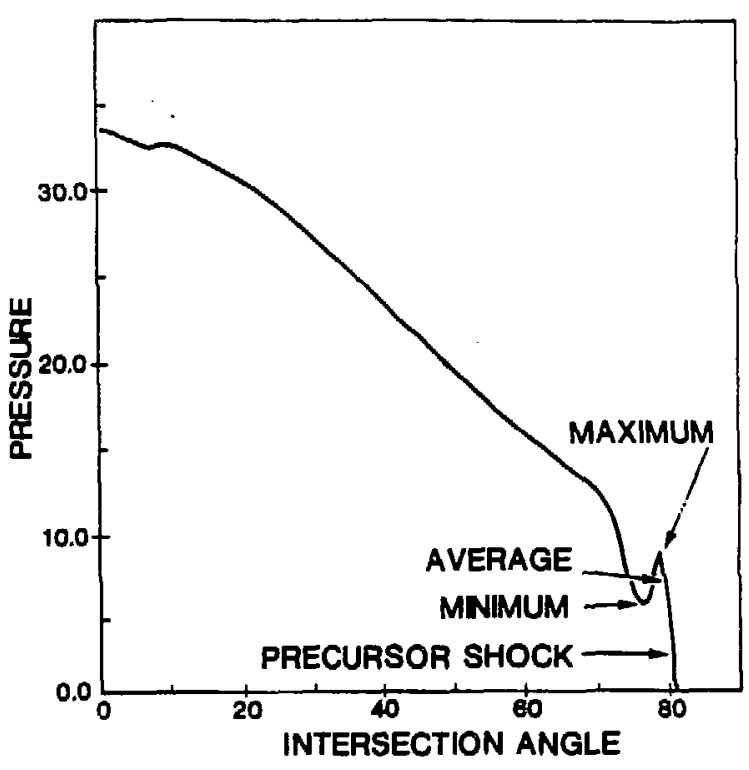

Fig. 20. Pressure as a function of intersection angle, showing the maximum and minimum pressures used to calculate the uncertalnty bars of Fig. 19. 

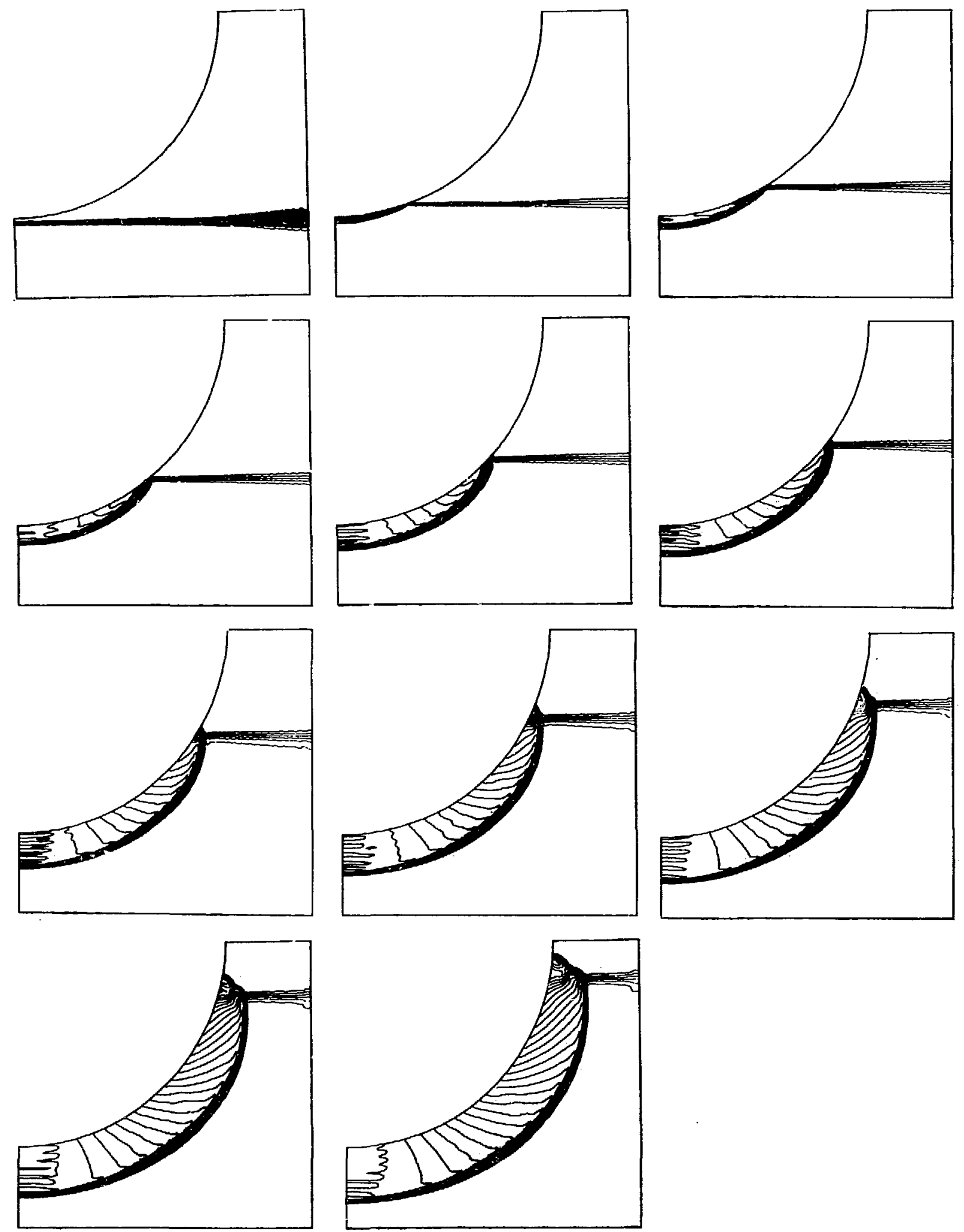

F1g. 21. The evolution of shock structure as an Incident shock $(M=3.11)$ passes over a rigid cylinder. 
A comparative calculation of this cylinder case with the new mesh, versus this cylinder case with the mesh used in the first cylinder case, shows that the results are very similar. The only deviation of results is that for large incident angles the precursor shock of FIg. 2018 smeared out by the less optimal zoning.

Figure 21 shows the evolution of the shock structure by means of pressure contours. This shows that at an intersection angle between 46 and 52 degrees the transformation from regular reflection to simple Mach refiection occurs. At large Intersection angles, a complex shock structure develops. These contour plots show the two pressure changes along the cyllnder wall, as shown on Fig. 19, at angles of Intersection greater than 60 degrees.

To simulate shock interaction around a rigld sphere we changed from planar geometry to cylindrical geometry. Th1s change makes the left vertical axis a cylindrical axis of symetry, thus simulating a sphere. The Input conditions we used for this spherlcal case are the same as those used in our second cylinder case.

Figure 22 plots $\Delta \mathrm{P}_{\mathrm{r}} / \Delta \mathrm{P}_{\text {Inc }}$ as a function of the intersection angle. Thls figure shown a comparison of the spherical case and its corresponding cylindrical case. The + points with circles around them are results from the cylindrical calculation and the + points wth no circle around them are results from the spherical calculation. The results of the two calculations are very simflar, but show that the pressures are lower In the spherical case than they are for the cylindrical case. This $1 \mathrm{~s}$ to be expected because the pressure can be relleved much faster around the sphere by lateral

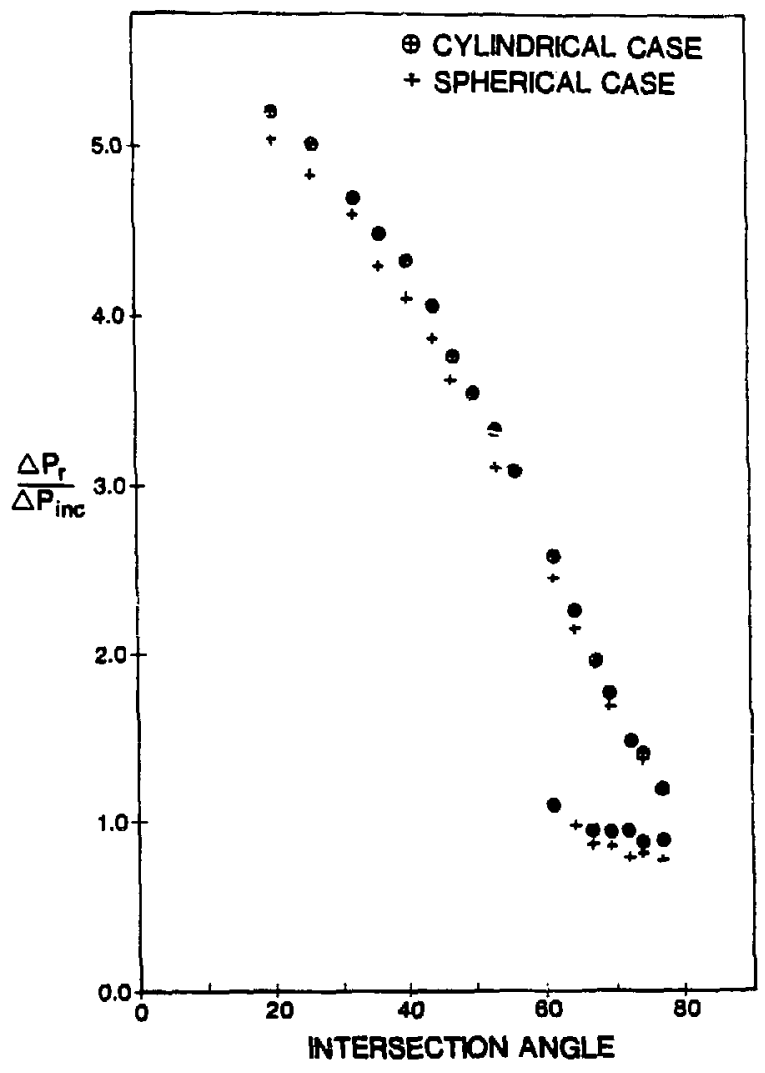

FIg. 22. $\Delta \mathrm{P}_{\mathbf{r}} / \Delta \mathrm{P}_{\text {Inc }}$ ag a function of intersection angle for cylindrical and spherical rigid obstacles; $M=3.11$. 
divergence of flow in all directions, whereas the cylindrical pressure can be relieved by divergence only in directions normal to the cylindrical axis.

Figure 23 shows the evolution of shock structure as the Incident shock passes over the rigid sphere. The features shown on these computer-generated contour plots are quite similar to those shown In Fig. 21 .

Another form of testing the calculational results is accomplished by theoretically calculating the reflected pressure of the incident shock initially hitting the cylinder and sphere and the steady-state pressure at that stagnation point. The behavior of the incident shock hitting the Initial impact position is briefly like that of an Incldent shock hitting a plane wall. We calculate the theoretical reflected pressure by placing the shock hitting the rigid wall in the framework that the fluid ahead 18 at rest, and applying the following equations [4]

$$
\begin{aligned}
& u=\frac{u_{-}}{c_{-}}, \text {where } c_{-}=\left[\frac{\gamma p_{-}}{\rho_{-}}\right]^{\frac{1}{2}}, \\
& M=\frac{\gamma+1}{4} u+\left[1+\left(\frac{\gamma+1}{4} u\right)^{2}\right]^{\frac{1}{2}}, \\
& P=\left[1+\frac{2 \gamma}{\gamma+1}\left(M^{2}-1\right)\right] .
\end{aligned}
$$

Knowing $P$ we can calculate the reflected pressure by multiplying it by $p_{-}$. 

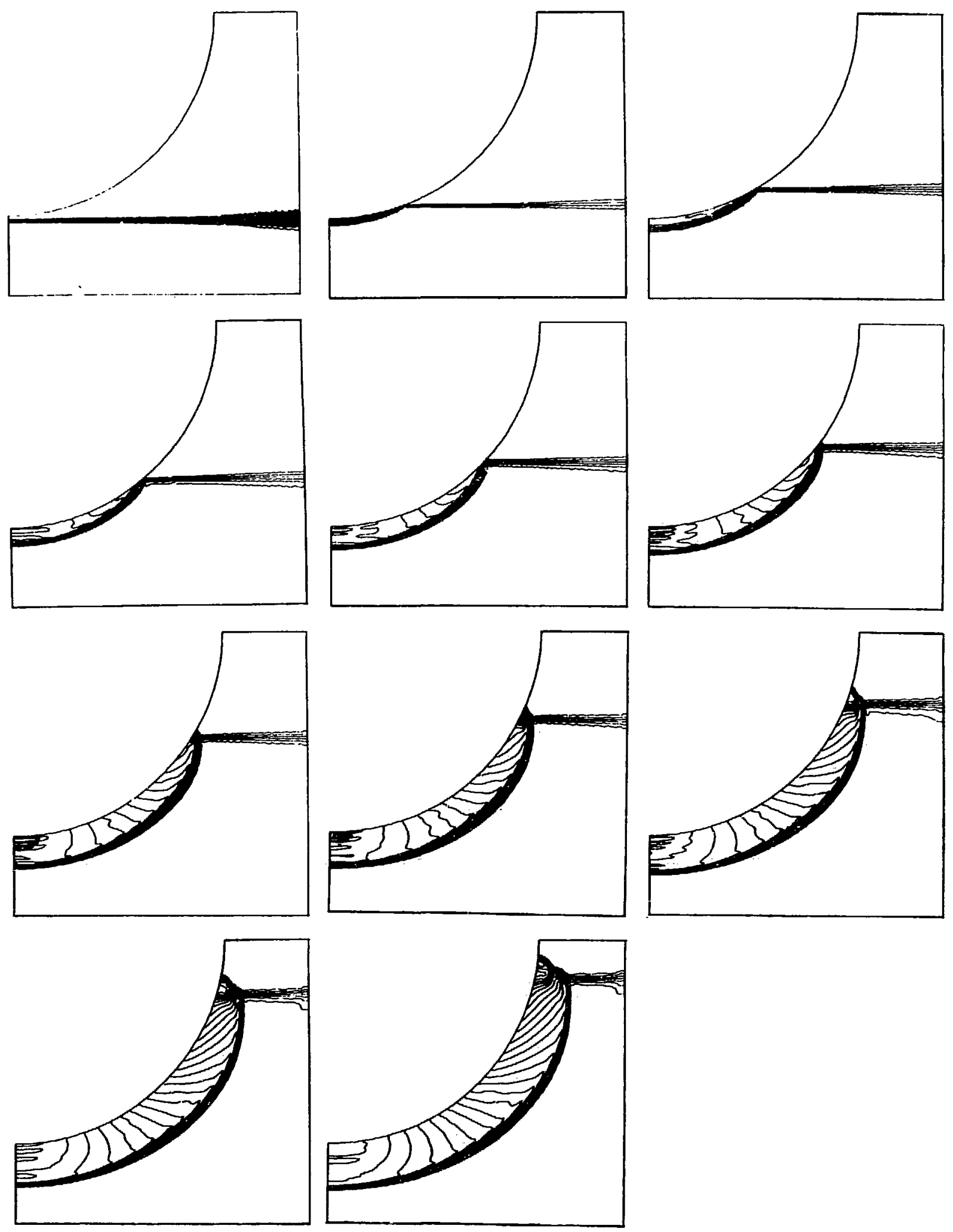

F1g. 23. The evolution of shock structure as an incident shock $(M=3.11)$ passes over a rigid sphere. 
Keeping ourselves in the framework where the fluid ahead is at rest we can calculate the theoretical steady-3tate pressure at the stagnation point $S$. By knowlng $p_{-}, p_{-}, I_{-}, u_{-}$, and $\gamma$ we can calculate $p_{+}, \rho_{+}, I_{+}$, and $u_{+}$using the following equations

$$
\begin{aligned}
M_{R S} & =\frac{u_{-}}{a_{-}}, \\
u^{\prime} & =\frac{2\left(H_{R S}^{2}-1\right)}{(\gamma+1) M_{R S}}, \\
p^{\prime}= & 1+\frac{2 \gamma}{\gamma+1}\left(M_{R S}^{2}-1\right), \\
Z^{\prime}= & -\frac{\gamma+1}{1+\left(\frac{2}{M_{R S}^{2}}\right)},
\end{aligned}
$$

where $M_{R S}$ is the Mach number of the reflected shock and

$$
\begin{aligned}
& P_{+}=p_{-} p^{\prime}, \\
& \rho_{+}=\rho_{-} z^{\prime}, \\
& I_{+}=\frac{p_{+}}{(\gamma-1) p_{+}}, \\
& u_{+}=c_{-} u^{\prime},
\end{aligned}
$$

in which $p_{+}, \rho_{+}, I_{+}$, and $u_{+}$are the pressure, density, Incernal energy, and materlal velocity across the reflected shock.

We now move back to the framework in which the cylinder is at rest. KnowIng these quantitles ( $p_{+}, p_{+}, I_{+}$, and $u_{+}$) we can follow a Bernoulli 1 ine and an adiabat to the stagnatlots point. The Bernoull 11 ne 18 as follows 


$$
\left(\frac{p}{p}+I+\frac{1}{2} \tilde{u}^{2}\right)_{+}=\left(\frac{p}{p}+I\right)_{g},
$$

rearranging this we get

$$
\left(\frac{p}{\rho}+\frac{\gamma-1}{2 \gamma} \tilde{u}^{2}\right)_{+}=\left(\frac{p}{\rho}\right)_{s} \text {. }
$$

The adiabat is $\left(\frac{p}{\rho \gamma}\right)_{+}=\left(\frac{p}{\rho}\right)_{s}$.

Thus solving (5) and (6) we obtain the theoretical stagnation pressure $\mathrm{p}_{8} \cdot \mathrm{Fig}$ ure 24 illustrates where the stagnation point $1 \mathrm{~s}$ and where the + quantities are calculated.

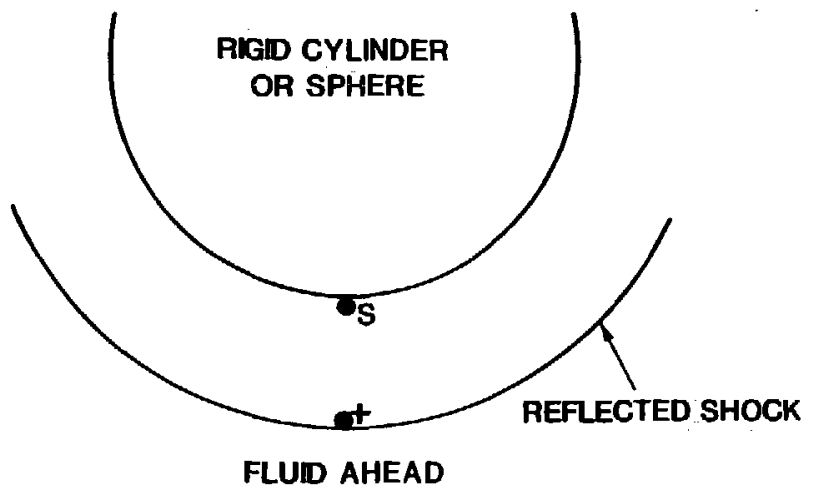

Fig. 24. Location of stagnation point, $s$. 
Figure 25 shows pressure at the stagnation point versus time for both the cylindrical and spherical cases. Time equal to zero corresponds to the time when the Incident shock hits the cylinder and sphere. Our spherical results agree at late times with the expected asymptotic stagnation pressure. The curve also extrapolates well towaras the theoretical inftial reflected pressure. Our curve for the cylinder also extrapolates well towards the theoretical initial reflected pressure. However, it approaches the theoretical stagnation asymptote much slower than the spherical curve. This is also due to the fact that the pressure at the spherical stagnation point can be relieved by lateral divergence of the flow in all directions away from the stagnation point. In contrast, the cylindrical stagnation pressure can be relleved by divergence only in directions normal to the cylindrical axis.

These results for the rigid cylinder and sphere show that CAVEAT has the capabllity to describe the complex process of shocks in gases interacting with rigld cylinders and spheres. When we compare our computational results to the experimental data (as In Figs. 17 and 19) we find that our computations are hIgher, as discussed in Sec. VI.

\section{DEFORHABLB CYLINDRICAL AID SPHERICAL SHBLLS}

Even though there are no experimental data avallable to make comparisons we nevertheless investigate shock interaction with deformable cylindrical and spher-

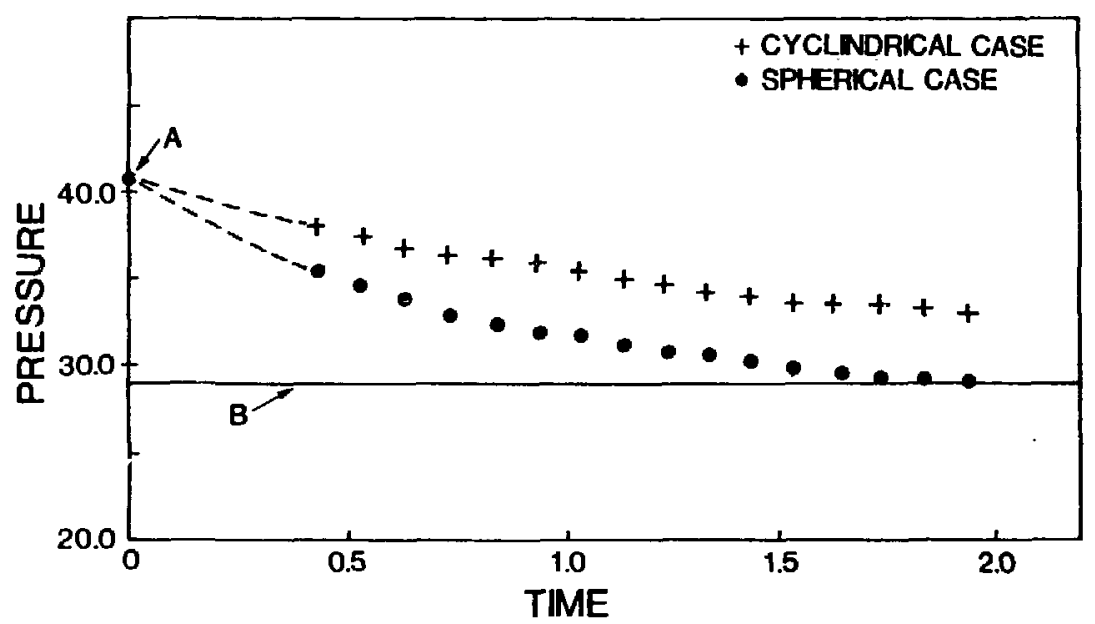

Fig. 25. Pressure as a function of time at the stagnation point. A 1s the theoretical initial pressure and $B$ is the theoretical stagnation asymptote. 
ical she11s. Here we are concerned with shell deformation, and with the changing shock structures and pressures generated by the shock Interaction with these deformable surfaces at continuously changing angles of intersection. The transmitted shock through the shell interacts with 1ts inner surface, which may be either free or a rigid wall. In the latter case we examine the transition from regular to Mach reflection at that surface.

Our logically rectangular mesh is simflar to the one shown in Fig. 18 except that we place a strip of mesh along the top of the logically rectangular mesh. This strip of mesh represents the deformable she11. This mesh consists of only one region with two parts; part one being the calculational mesh used in our rigid calculations and part two belng the deformable shell. We require specifying only part of the bottom logical side as inflow. Flgure 26 shows the logical1y rectangular mesh and the corresponding computational mesh used in these studies.

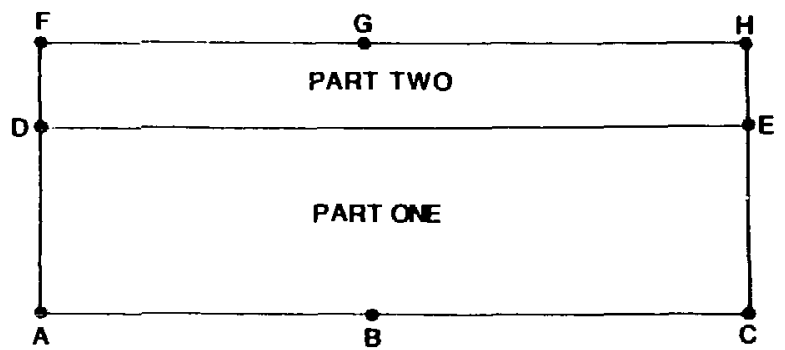

Fig. $26(a)$

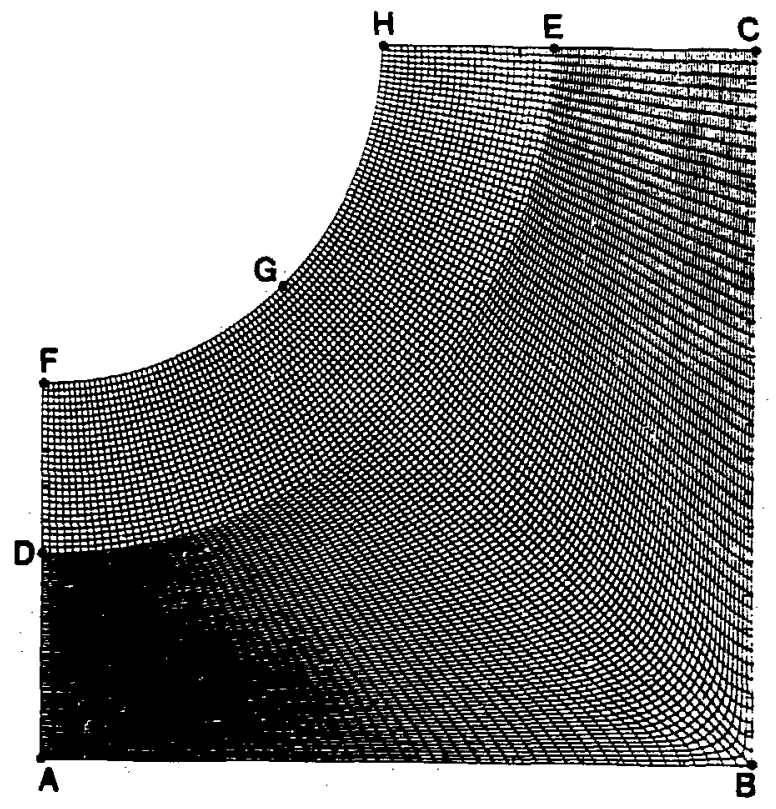

Fig. $26(b)$

Fig. 26. (a) configuration of the logically rectangular mesh used in the cylindrical and spherical shell problem; A to B is inflow; C to $H$ is outflow; $A$ to $F$ is elther reflective or a cylindrical axis; $B$ to $C$ is reflective; $F$ to $\mathrm{H} 1 \mathrm{~s}$ elther reflective or free; $D$ to $\mathrm{E}$ 1s Lagrangian; part two is the shell region and (b) configuration of the computational mesh. 
In order to run these types of problems correctly, we need to use the adaptive mesh technique in the CAVEAT code. This enables the interface to move and our mesh to adapt to the motion of the interface. The outer shell boundary (1.e., the interface between materials) is treated as Lagrangian in the normal direction of its motion. Table IV lists the Input conditions for the deformable series.

\section{TABLE IV}

INPUT DATA FOR THE DEFORMABLE CYLINDRICAL SPHERICAL, AND CONICAL SHELL CALCULATIONS

\begin{tabular}{|c|c|c|c|c|c|c|c|c|c|c|}
\hline $\begin{array}{l}\text { Figure } \\
\text { Number }\end{array}$ & $\begin{array}{c}\text { Run } \\
\text { Number }\end{array}$ & $\mathbf{M}$ & $\mathbf{V}_{\mathbf{s}}$ & $\rho-$ & p. & u. & $\rho_{S H}$ & $\mathbf{p}_{S \mathrm{H}}$ & Ys & $\begin{array}{l}\text { Interior } \\
\text { Boundary }\end{array}$ \\
\hline $26,28,29$ & $\begin{array}{l}\text { DEFCYCS7 } \\
\text { DEFCYCS7A }\end{array}$ & 3.11 & 3.11 & 3.96 & 7.94 & 2.32 & 1000 & 0.714 & 1.4 & Rigid \\
\hline 30,31 & $\begin{array}{l}\text { DEFCYCSB } \\
\text { DEFCYCSBA }\end{array}$ & 3.11 & 3.11 & 3.96 & 7.94 & 2.32 & 2.0 & 0.714 & 1.4 & Rigid \\
\hline 32 & $\begin{array}{l}\text { DEFCYCS9. } \\
\text { DEFCYCS9A }\end{array}$ & 3.11 & 3.11 & 3.96 & 7.94 & 2.32 & 2.0 & 0.714 & 1.4 & $\begin{array}{l}\text { Applied } \\
\text { Pressure }\end{array}$ \\
\hline 34 & $\begin{array}{l}\text { DEFSPS10 } \\
\text { DEFSS10A }\end{array}$ & 3.11 & 3.11 & 3.96 & 7.94 & 2.32 & 1000 & 0.714 & 1.4 & Rigid \\
\hline 35,36 & $\begin{array}{l}\text { DEFSPS11 } \\
\text { DEFSS11A }\end{array}$ & 3.11 & 3.11 & 3.96 & 7.94 & 2.32 & 2.0 & 0.714 & 1.4 & Rigid \\
\hline 37 & $\begin{array}{l}\text { DEFSPS } 12 \\
\text { DEFSS } 12 A\end{array}$ & 3.11 & 3.11 & 3.96 & 7.94 & 2.32 & 2.0 & 0.714 & 1.4 & $\begin{array}{l}\text { Applied } \\
\text { Pressure }\end{array}$ \\
\hline 39,40 & DEFWDG 1 & 3.36 & 3.36 & 4.16 & 9.29 & 2.55 & & & & Rigid \\
\hline 38 & DEFWDG2 & 3.36 & 3.36 & 4.16 & 9.29 & 2.55 & 2.0 & 0.714 & 1.4 & $\begin{array}{l}\text { Applied } \\
\text { Pressure }\end{array}$ \\
\hline $42,43,46$ & DEFWDG3 & 3.36 & 3.36 & 4.16 & 9.29 & 2.55 & 2.0 & 0.714 & 1.4 & $\begin{array}{l}\text { Applied } \\
\text { Pressure }\end{array}$ \\
\hline $47,48,49$ & DEFWDG4 & 3.36 & 3.36 & 4.16 & 9.29 & 2.55 & 5.0 & 0.714 & 1.4 & $\begin{array}{l}\text { Applied } \\
\text { Pressure }\end{array}$ \\
\hline & DEFWDG5 & 3.36 & 3.36 & 4.16 & 9.29 & 2.55 & 20.0 & 0.714 & 1.4 & $\begin{array}{l}\text { Applied } \\
\text { Pressure }\end{array}$ \\
\hline $44,45,46$ & DEFWDG6 & 3.36 & 3.36 & 4.16 & 9.29 & 2.55 & 0.5 & 0.714 & 1.4 & $\begin{array}{l}\text { Applied } \\
\text { Pressure }\end{array}$ \\
\hline 50,51 & DEFWDG7 & 3.36 & 3.36 & 4.16 & 9.29 & 2.55 & 10.0 & 0.714 & 1.4 & $\begin{array}{l}\text { Applied } \\
\text { Pressure }\end{array}$ \\
\hline
\end{tabular}


We first experiment with the deformable cylindrical and spherical shells by making the density in the shell large $\left(\rho_{\mathrm{SH}}=1000.0\right)$ so that we can simulate an almost rigid case to compare results to our rigid case. We specify the initial pressure inside the shell equal to the initial pressure outside so that we keep the system in equilibrium. Pre-shock and the post-shock conditions are chosen to simulate the second rigid cylinder problem, with $M=3.11$, previously discussed. For each of our deformable objects we use for conventence a polytropic-gas equation of state. In general, many CAVEAT runs utilize table-look-up equations of state for a variety of real materials.

Figure 27 shows a comparison of $\Delta P_{r} / \Delta P_{\text {inc }}$ as a function intersection angle for our almost rigid cylindrical shell calculation and our rigld cylinder calculation. The dot points are from our almost rigld calculation and the + points are the comparison results from our rigid cylinder calculation. This comparison shows that our almost rigid calculation is behaving very similar to our rigid calculation. We see that at small intersection angles the pressures from our almost rigid calculation are lower than the pressures from our rigid calculation, because our deformable cylindrical shell furnishes a relief in pressure. The precursor shock observed at large angles for the rigid cylinder likewise occurs for the deformable cylinder. These pressures are shown in this figure without the uncertainty bars as in Fig. 19. Figure 28 shows the initial interface position and the slightly deformed interface position after the incident shock hits. We notice, however, that at large Intersection angles the pressure around our deformable cylinder is slightly higher than for our rigid cylinder calculation. This is probably because using an adaptive mesh in these calculations gives us better resolution around the shock structure than using an Eulerian mesh in our rigid cylinder calculations. Figure 29 shows this slightly better resolution of shock structure, in comparison with the resolution shown in Fig. 21 . These results indicate that CAVEAT is able to handle a large density difference between adjacent materlals. They also exhibit consistent results using very different approaches such as rigid versus almost rigid, and Eulerian mesh versus an adaptive mesh.

We now Investigate a larger deformation of the shell by making the density in the shell much smaller. Keeping all the Initial conditions the same as above, we make the density of the cylindrical shell equal to 2.0 , which is twice the initial density in the surrounding material. In this case we keep the inner boundary of the shell rigid. 


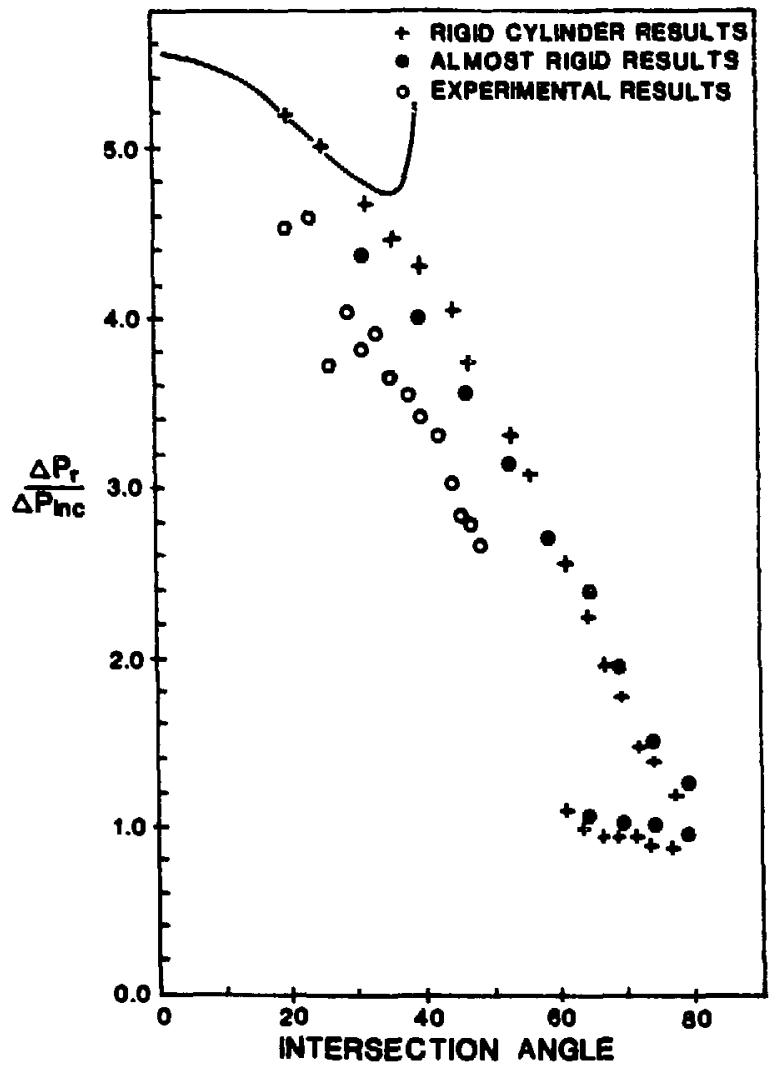

F1g. 27. $\Delta P_{r} / \Delta P_{\text {Inc }}$ as a function of intersection angle for $M=3.11$.

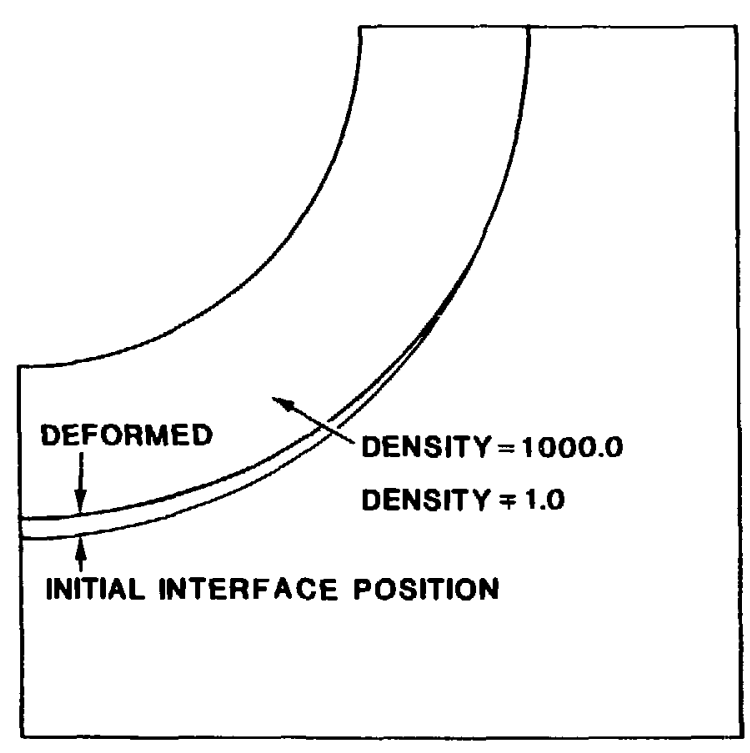

Fig. 28. Configuration of initial interface position with deformed interface of the almost rigid shell.

Figure 30 shows the evolution of shock structure as the incident shock travels past the deformable cylindrical she11. After the incident shock hits, a transmitted shock emerges Inside the more dense shell. This transmitted shock lags slightly behind the incident shock. We see the deformation of the interface, which 18 represented by the dashed line between the transmitted and the reflected shocks. When the transmitted shock hits the rigid inner boundary it produces a second reflected shock. Figure 31 shows the Interface position at various times. The Incident shock location can be Identified by locating the position where the interface first begins to deform. At the last time shown, the interface has a kink in it where part of the Interface appears to be parallel to the Inner boundary. The position of this kink corresponds to the Intersection with the reflected shock from the inner boundary. 

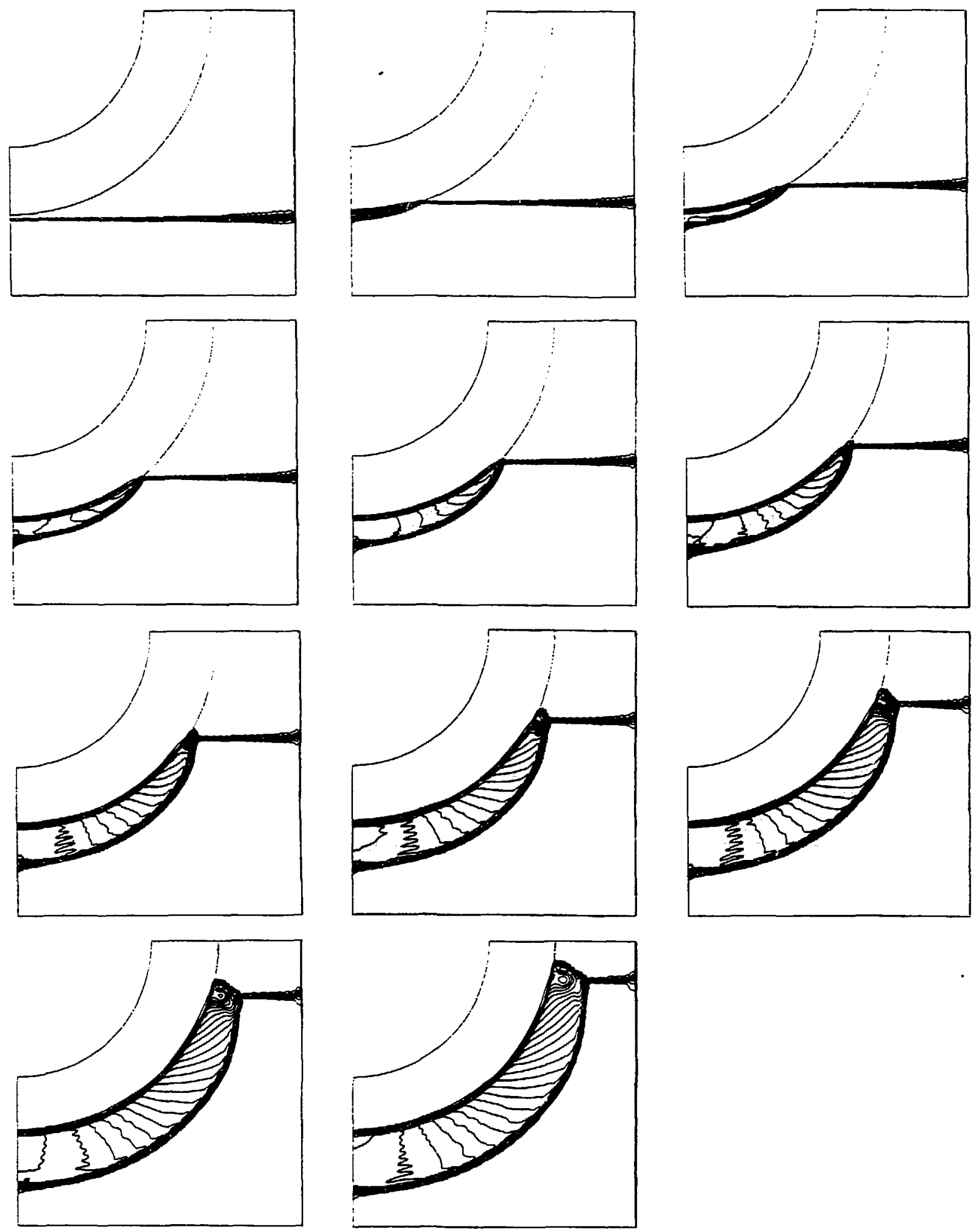

Fig. 29. The evolution of shock structures as an incident shock ( $M=3.11)$ passes over an almost rigld cylinder. 

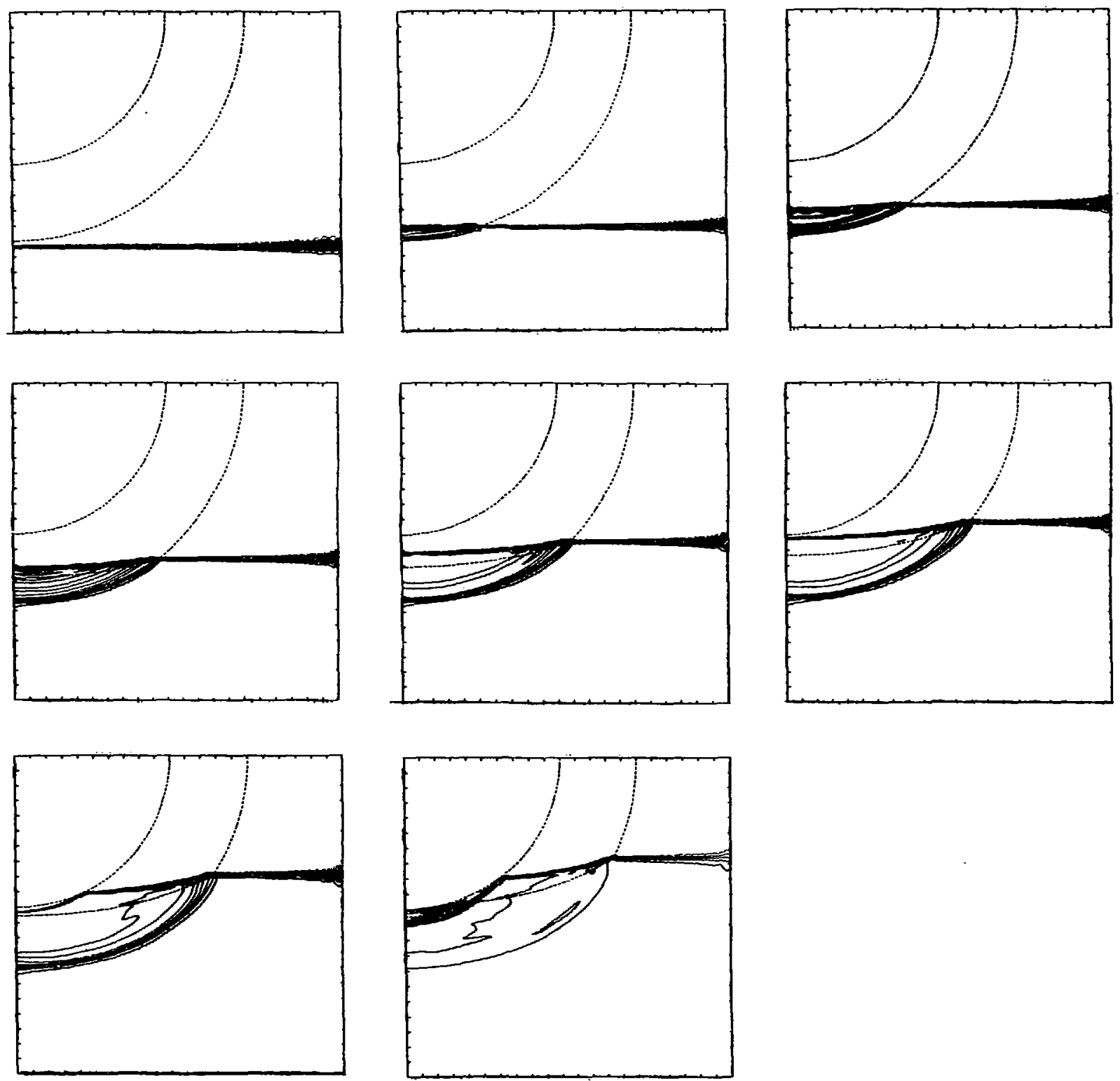

Fig. 30. The evolution of shock structure as an incident shock $(M=3.11)$ passes over a deformable cylindrical shell $\left(\rho_{S H}=2.0\right)$ with a rigid interior boundary. 


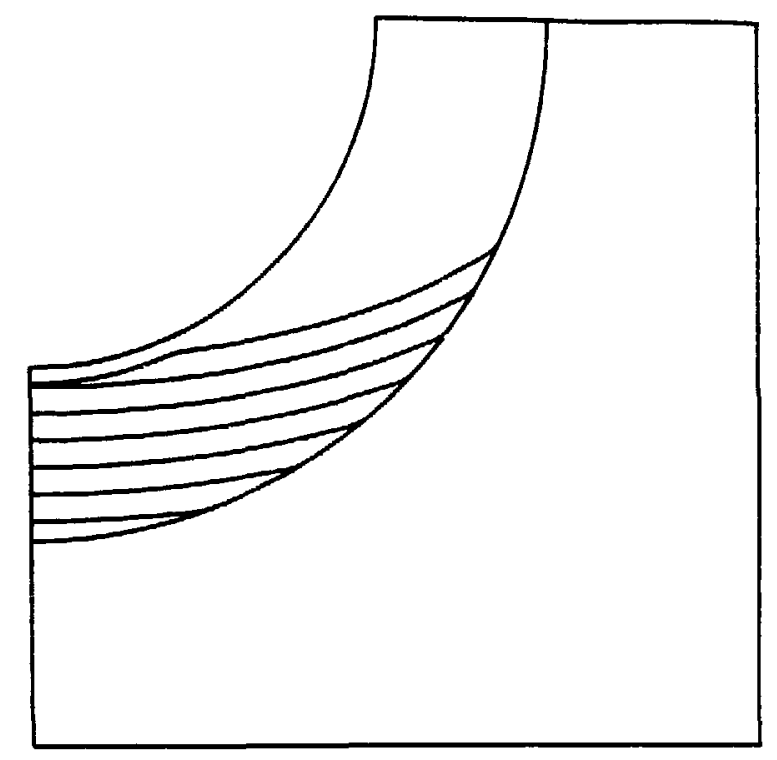

Fig. 31. Interface position at a sequence of times.

By making the Inner surface an applied pressure surface with an applied pressure specified such that it gives us initial equilibrium, we investigate the effects of the transmitted shock hitting a deformable inner boundary rather than a rigid one. Figure 32 shows the evolution of shock structure and the deformation of the interface and the inner boundary. The parameters for this calculation are identical to those of the previous case with the exception of the inner boundary treatment. Figure 32 shows that as the transmitted shock hits the Interior boundary the boundary bulges in towards the center of the cylinder and a strong rarefaction is reflected. Simple Mach reflection develops at the outer interface of the cylindrical shell as the angle of intersection becomes larger.

To convert the geometry of these calculations to represent spherical shells we use cylindrical coordinates in which the left axis 18 a cylindrical axis. To simulate an almost rigid sphere we make the density in the shell region equal to 1000.0. The initial conditions for this slightly deformable spherical shell problem are the same as the conditions used for the almost rigid cylindrical shell case. Using our computational results of this problem we can determine $\Delta \mathrm{P}_{\mathbf{r}} / \Delta \mathrm{P}_{\text {inc }}$ and compare these results to the almost rigid cylindrical shell results. 

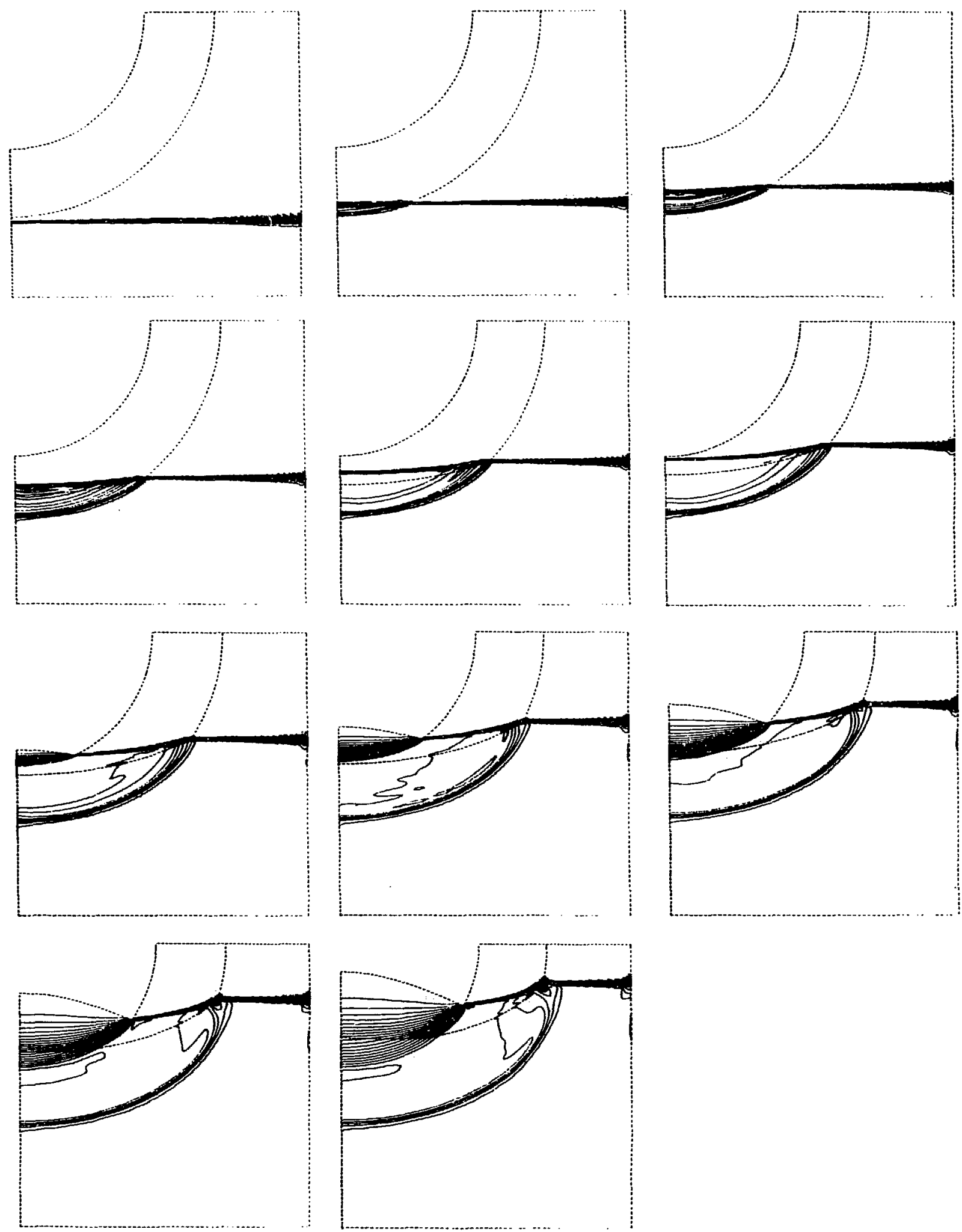

F1g. 32. The evolution of shock structure as an incident shock $(M=3.11)$ passes over a deformable cylindrical shell $\left(\rho_{\mathrm{SH}}=2.0\right)$ with the Inner boundary spectified as a free surface. 
Flgure 33 presents a comparison. The dot points refer to the cylirdrical case and the + points refer to the spherical case. The spherical pressures are again lower than the cylindrical pressures. Figure 34 1llustrates the evolution of shock configuration. A comparison of this figure with Fig. 27 shows that the shock evolution for both cases is quite simflar. A Mach stem forms at an intersection angle of about 50 degrees.

For a more deformable spherical shell we change 1ts density to 2.0. The Inner shell boundary is rigid, and the Mach number of the Incoming shock 18 3.11. Figure 35 showa the evolution of shock structure as the Incident shock passes over the deformable sphere. Th1s figure is similar to Fig. 30, which 11lustrates the shock passing over a cylindrical shell. At the interior boundary double Mach reflection occurs. Figure 36 glves a close-up view of this double Mach reflection. The reflected shock from the outer interface shows a simple Mach configuration; the strength of this shock 18 very weak compared to the transmitted shock and the reflected shock.

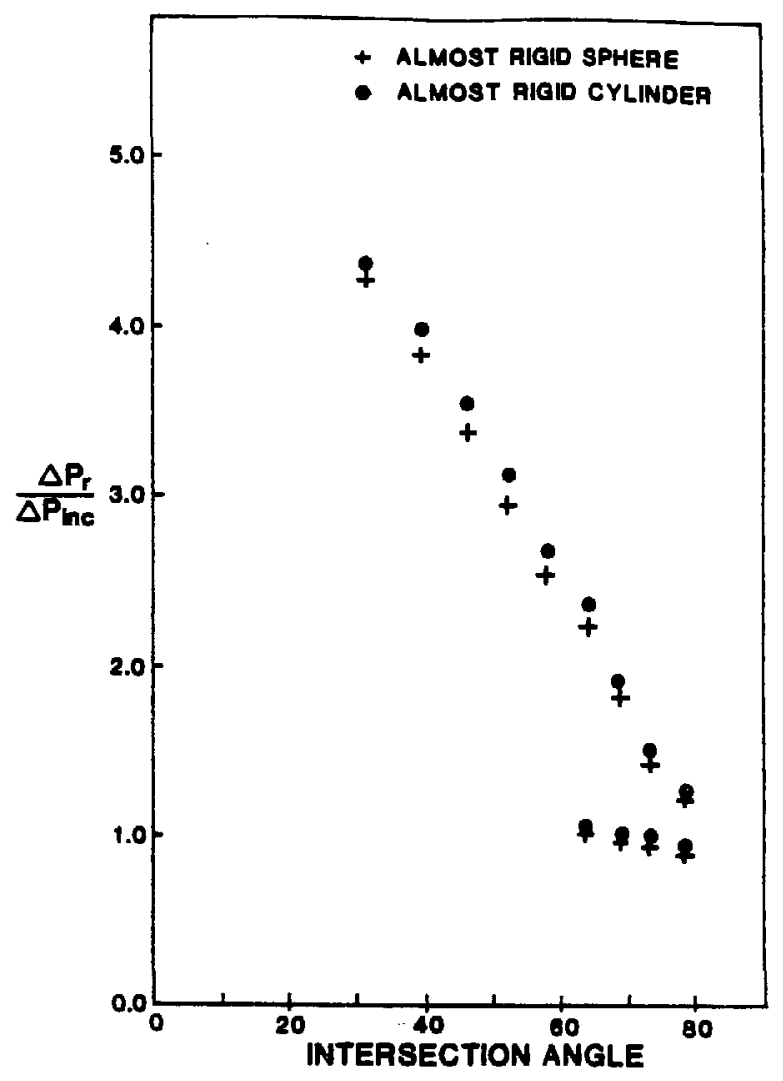

F1g. 33. $\Delta P_{r} / \Delta P_{\text {Inc }}$ as a function of intersection angle. 

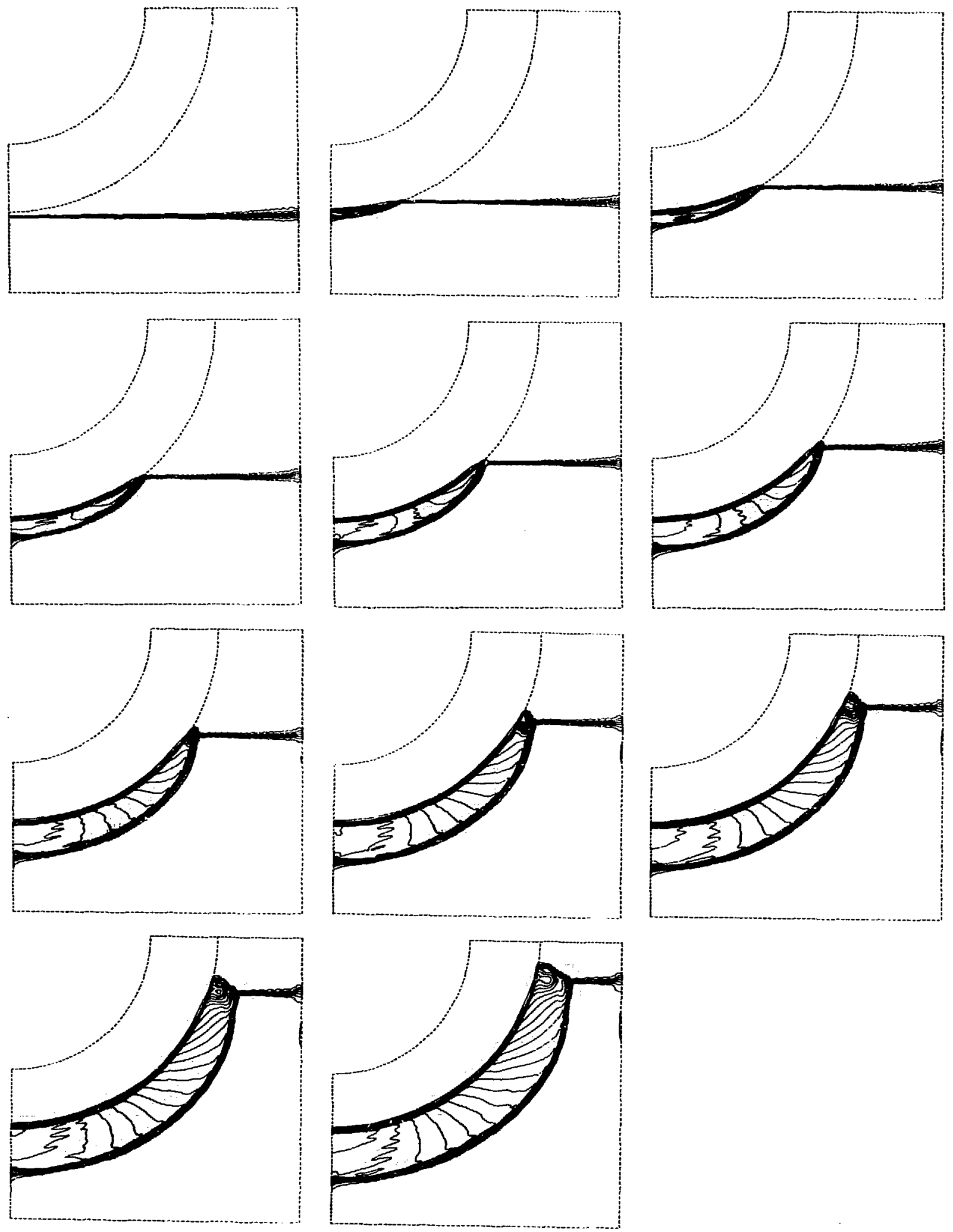

F1g. 34. The evolution of shock stcucture as an incident shock $(M=2=11$ ) passes over an almost rigid sphere $\left(p_{\mathrm{SH}}=1000.0\right)$. 

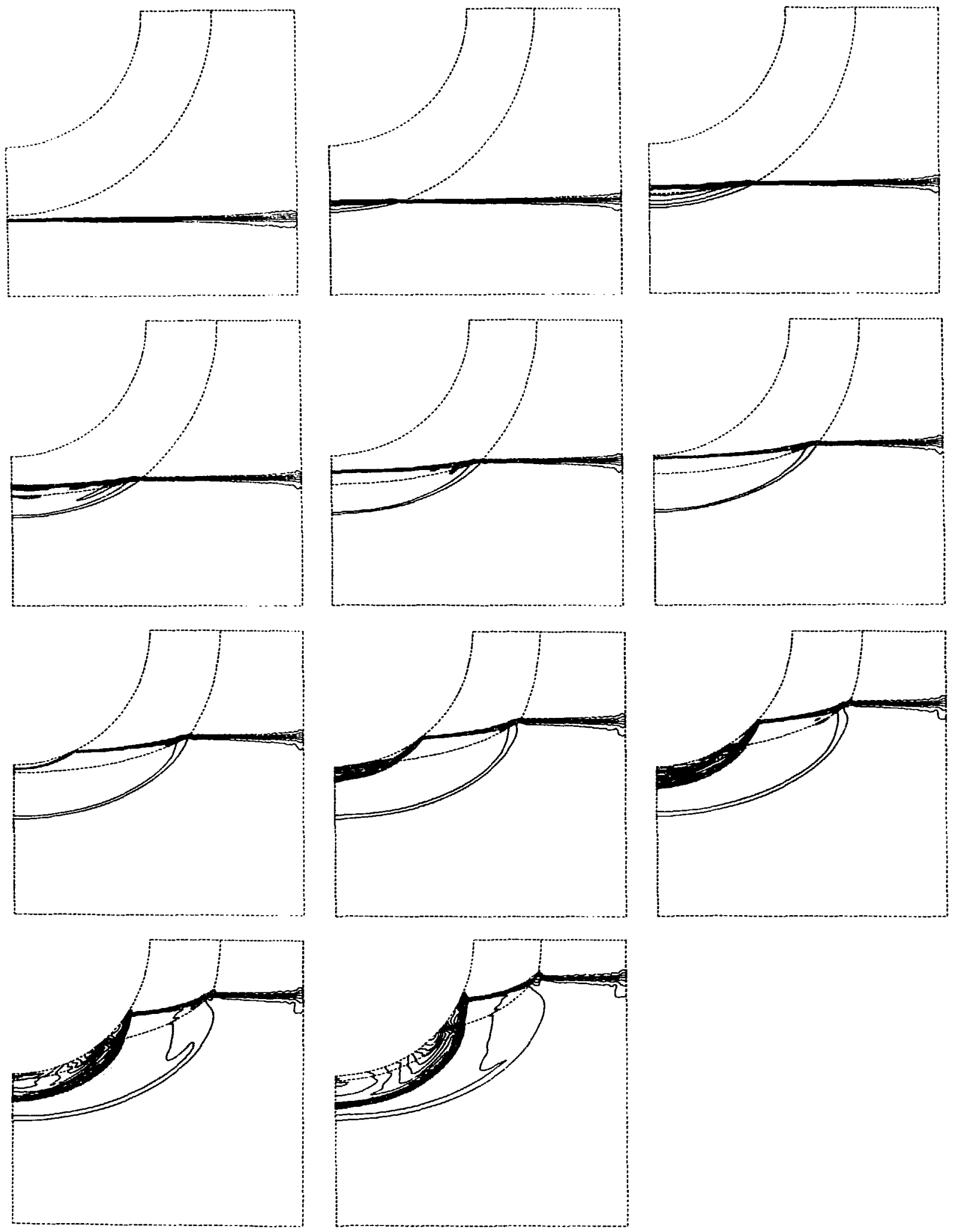

F1g. 35. The evolution of shock structure as an incident shock ( $M=3.11$ ) passes over a deformable spherical shell $\left(\rho_{\mathrm{SH}}=2.0\right)$ with rigit interior boundary. 
For the next calculation we exhiblt the deformation of the interior boundary by making it an applied pressure boundary. The applied pressure is equal to the intial pressure in the deformable shell and in the pre-shocked region surroundIng the shell, thus giving equilibrium. The shock evolution for this calculation Is very gimilar to that of the cylindrical she11 (see Fig. 32). As the transmitted shock hits the Interior interface, the interface bulges toward the center of the spherical shell. Flgure 37 shows a close-up view of the shock structure and Interface deformation at the final state of the calculation. With the angle of Intersection being large at this time, a Mach stem 18 present on the outer Interface. We see the strong rarefaction produced by the interaction of the transmitted shock with the interior interface.

These results for the deformable cylindrical and spherical she11 cases give insight into the capabilities of the CAVEAT code. By simulating almost rigid cyIndrical and spherical shells, we show that two very different treatments can produce similar solutions, demonstrating CAVEAT's capability for handling large density differences between materials. We also have illustrated CAVEAT's ability to handle deformation by means of an adaptive mesh technique.

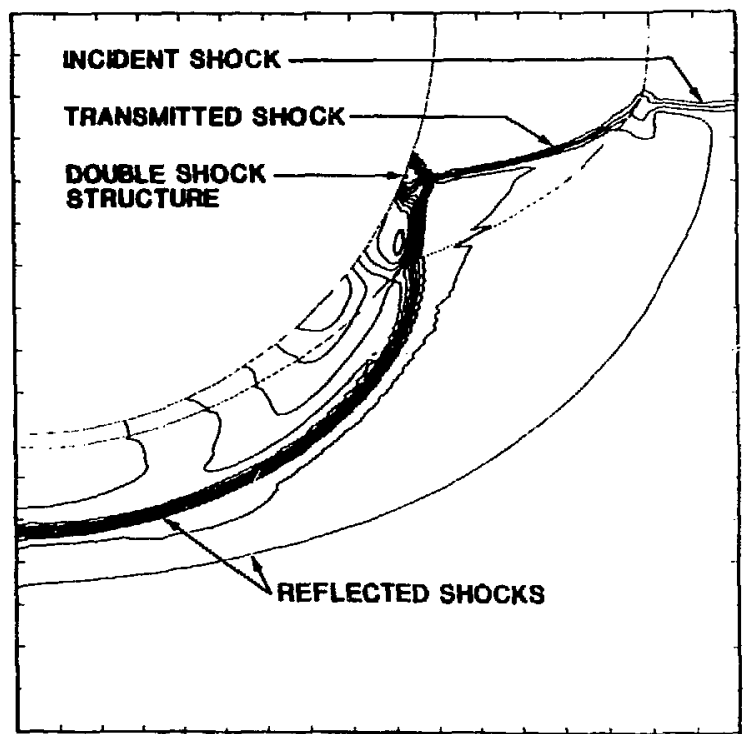

F1g. 36. A close-up view of Fig. 35 at a late time. Note: the contour interval in this figure is not the same as those in Fig. 35 .

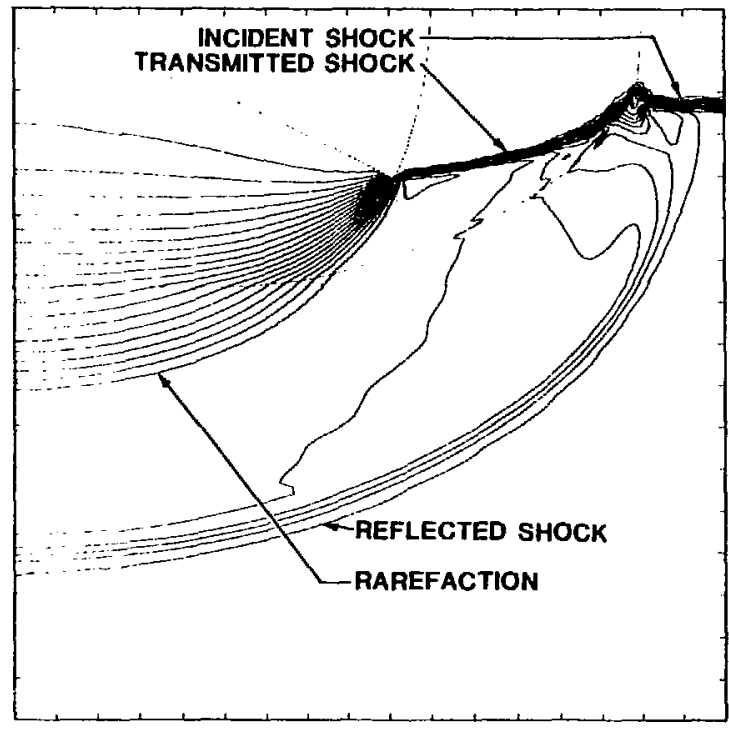

Fig.37. A close-up view of the shock structure at a late time as an incident shock passes over a deformable spherical shell $\left(_{S H}=2,0\right)$ with the Interior boundary being free. 


\section{DEFORLABLE CONICAL SHELLS}

The configuration for the conical shell calculations is similar to that for the wedge problems, with two differences: the tip of the conical shell curves and Intersects the left boundary at an angle normal to that boundary, and the left boundary is a cylindrical-coordinate axis. Figure 38 shows the computational mesh. We have used the same type of logically rectangular mesh as shown in Fig. 26. Instead of deforming the top boundary at a specific radius, we have deformed it as if we were making an oblique wedge. Then we have bent the tip of the wedge such that the interface is normal to the left boundary. The angle that the shell makes with the cylindrical axis is 43 degrees.

As a first step we ran a problem with the computational mesh shown in Fig. 39, In which the she11 has been replaced by a rigid wall, and planar geometry has been used to produce a blunt-nose wedge. The boundary conditions are as follows: bottom Is Inflow; top is outflow; right, left, and the obstacle are reflective. Using these boundary conditions and mesh we perform a run similar to our first rigld-wedge case. Figure 40 shows computer-generated contour plots of pressure and density. These exhfbit the double shock structure that the first wedge calculations indicate. Figure 41 compares $\Delta \mathrm{P}_{\mathbf{r}} / \Delta \mathrm{P}_{\text {Inc }}$ for this case wich results from

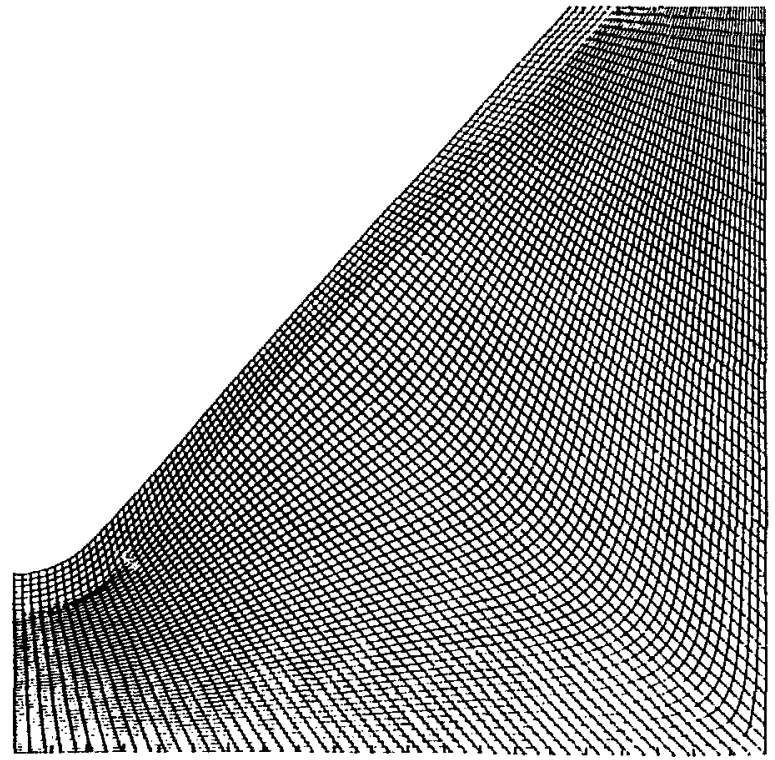

Fig. 38. The computational mesh used for the deformable conical shell studies.

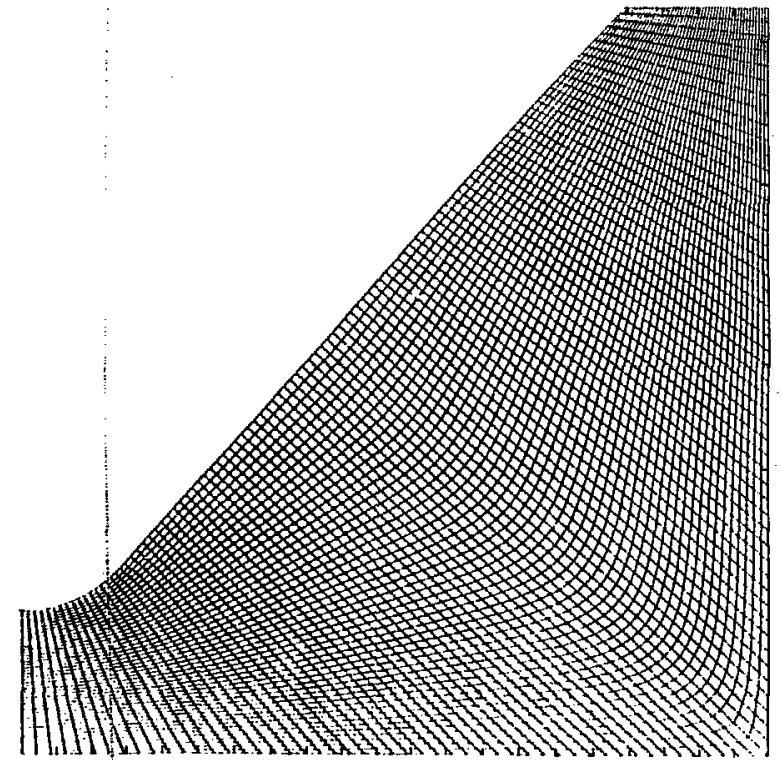

Fig. 39. The computational mesh used to simulate the first rigid wedge problem. 
the first wedge problem, and the experimental data of Helilg and Reichenbach. The two points plotted with circles around them are from the blunt-wedge computatlonal results. The solid curves are two- and three-shock theory and the circles with error bars are the experimental data. With the slightly different wedge shape in this calculation, our computational results nevertheless agree well with the experimental data.

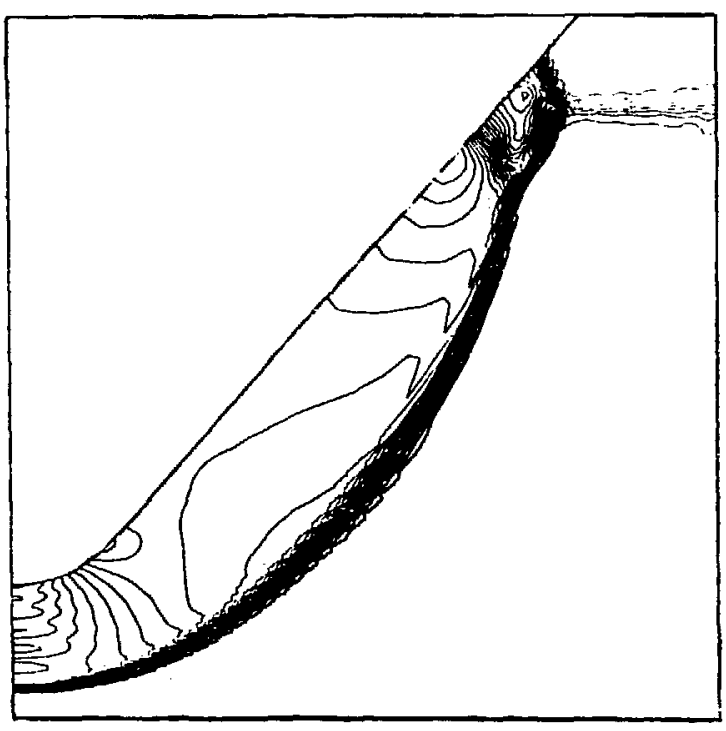

F1g. 40 (a)

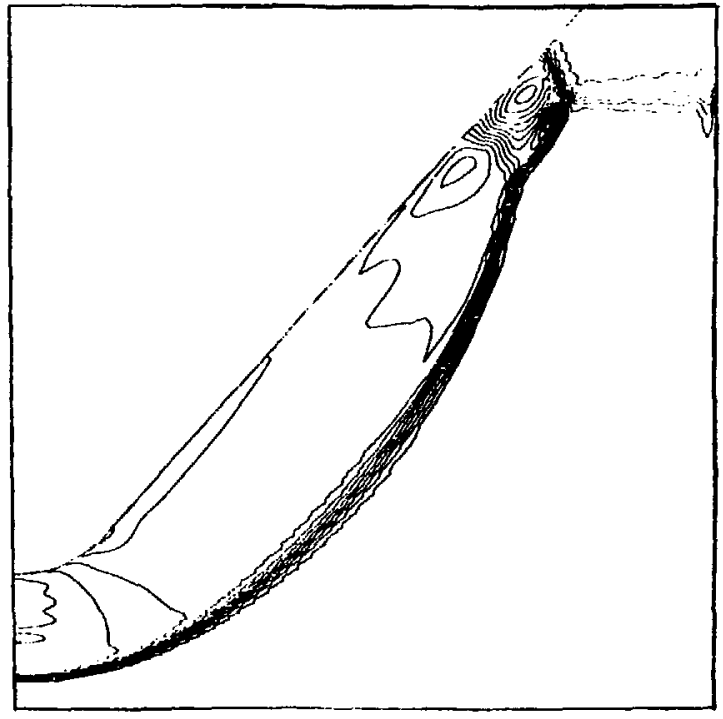

Fig. $40(b)$

FIg. 40. Computer-generated depictions of the blunt-nosed rigid-wedge problem using the mesh of $\mathrm{Fig}$. 39. (a) pressure contours and (b) density contours.

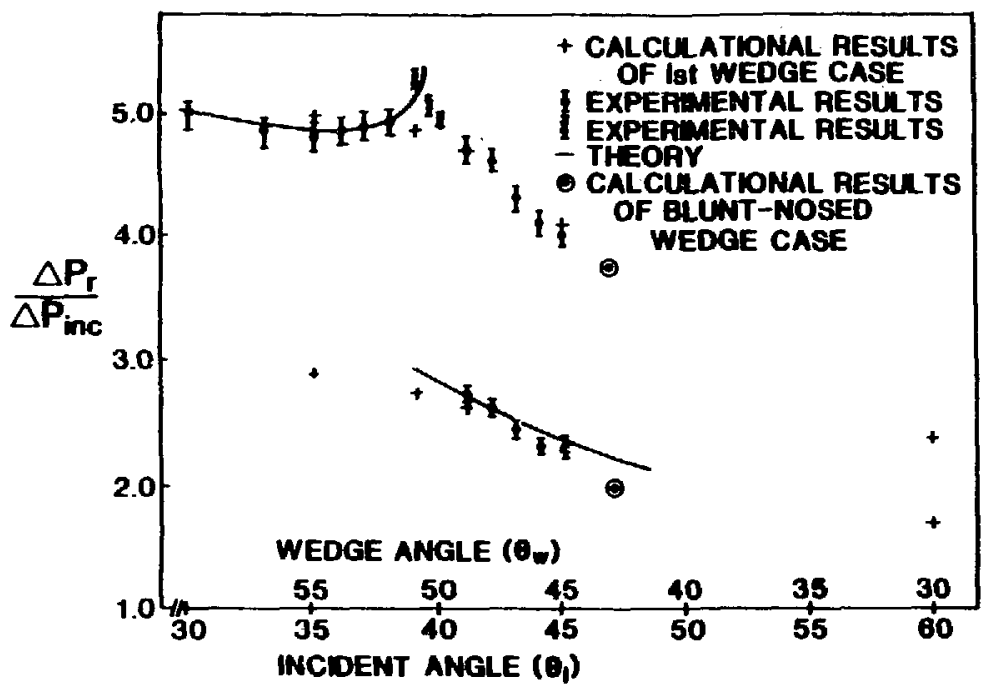

Fig. 41. $\Delta \mathrm{P}_{\mathrm{r}} / \Delta \mathrm{P}_{\text {Inc }}$ as a function of Iricident angle. 
Using the mesh shown In FIg. 38 and cylindrical geometry (left boundary an axis of symmetry) we investigate the deformation of a conical shell. This requires spectfying the interfor shell boundary as an applied pressure boundary and applying a pressure to give Initial equilibrium. Keeping all input conditions the same as the blunt-nosed wedge calculation we specify the InItial shell density equal to 2.0 and its Initial pressure equal to the Initial pressure surroundIng the shell. To handle the shell deformatirn we use the adaptive mesh. Figure 42 shows the evolution of shock structure and the deformation of the she11 at a sequence of times using computer-generated pressure contour plots. The interaction of the incident shock with the outer interface produces reflected and transmitted shocks. The transmltted shock then hits the interfor interface producing an Inversion of the tip of the conical shell. A strong rarefaction is also produced after the transmitted shock intersects with the interlor boundary. Figure 43 shows a time evolution of Interface deformation at the same times chosen for Fig. 42. The Incoming Incident shock position can be located by finding where the outer interface of the cone starts to deform. We find that the shell tip accelerates to a constant velocity, in this case approximately 1.5 times the shock velocity. Note that the central blob of material is hollow except for intersection of the leading edge with the axis. Beyond this last configuration it is expected that the entire flow fleld will propagate in an essentially self-similar fashion.

For a contrasting calculation we change the inftial density in the conical shell from 2 to 0.5 , such that the incident shock hits a lighter material. All the other Initial and Input conditions are the same as in the previous conical shell problem. Figure 44 shows the evolution of shock structure and the deformation of the shell at selected times. We see that as the incident shock hits the outer interface it produces a rarefaction rather than a reflected shock and a transmitted shock. In this case the transmitted shock moves ahead of the inc1dent shock so that the transmitted shock hits the interior interface more quickly than in the previous problem. Since the density of the shell is much smaller in this case the interface bulges up the axis of symmetry of the conical shell at a larger velocity (approximately 1.72 times the incident shock speed) than the case with the larger she1l density. Figure 45 shows the she11 position at the same times as in Fig. 44. Figure 46 shows a comparison of the shell position at the same time in these two cases. The kink structure seen in the shell with the larger density is not as severe in the lighter shell. 

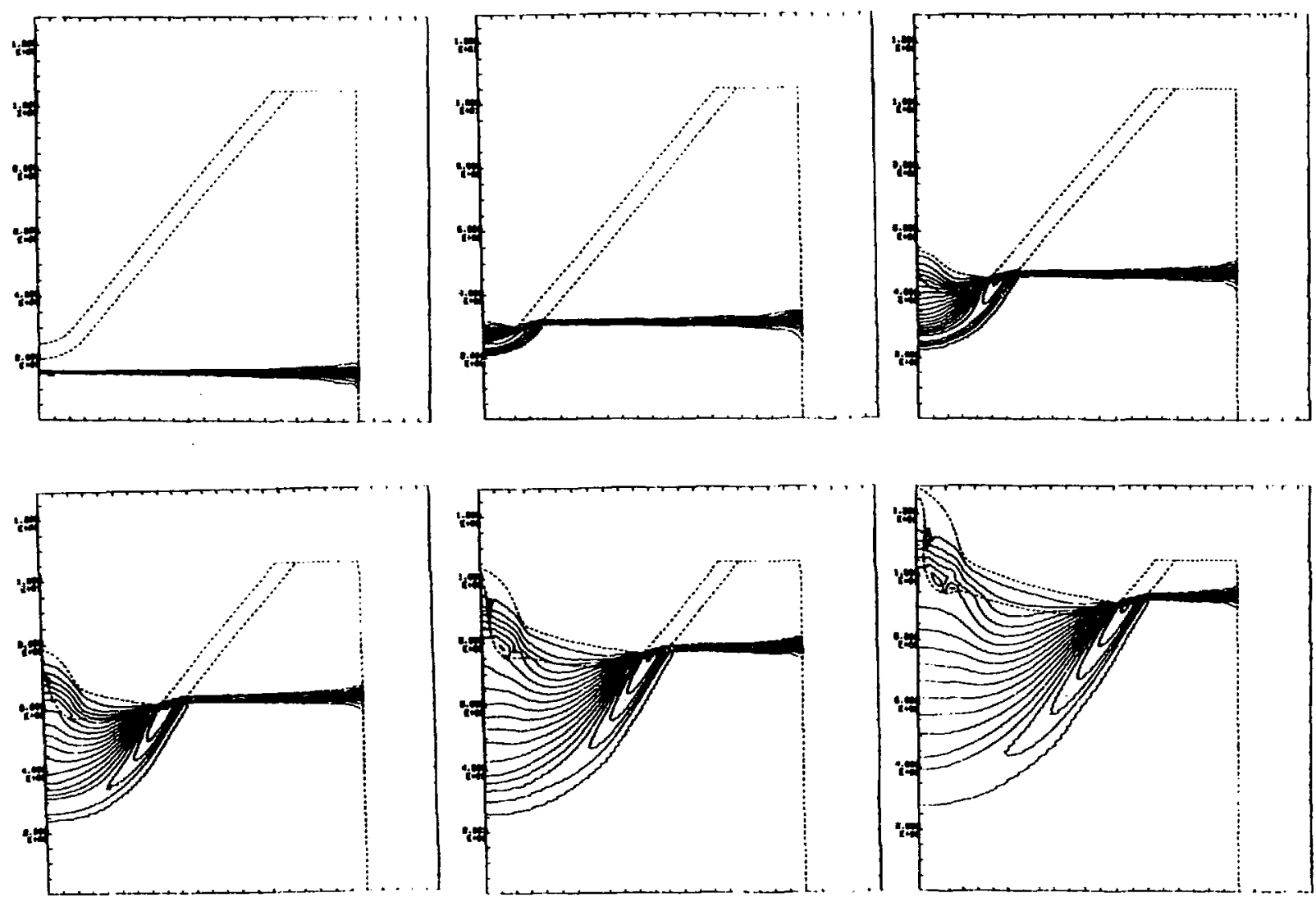

Fig. 42. The evolution of shock structure as an incident shock $(M=3.11)$ passes over a deformable confcal shell $\left(\mathrm{O}_{\mathrm{SH}}=2.0\right)$.

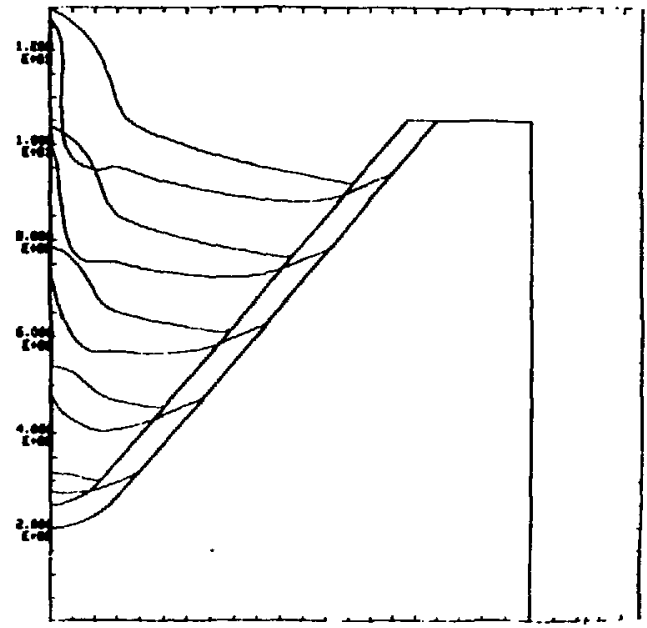

FIg. 43. Shell deformation for the problem of FIg. 42 at the same times as in that figure. 

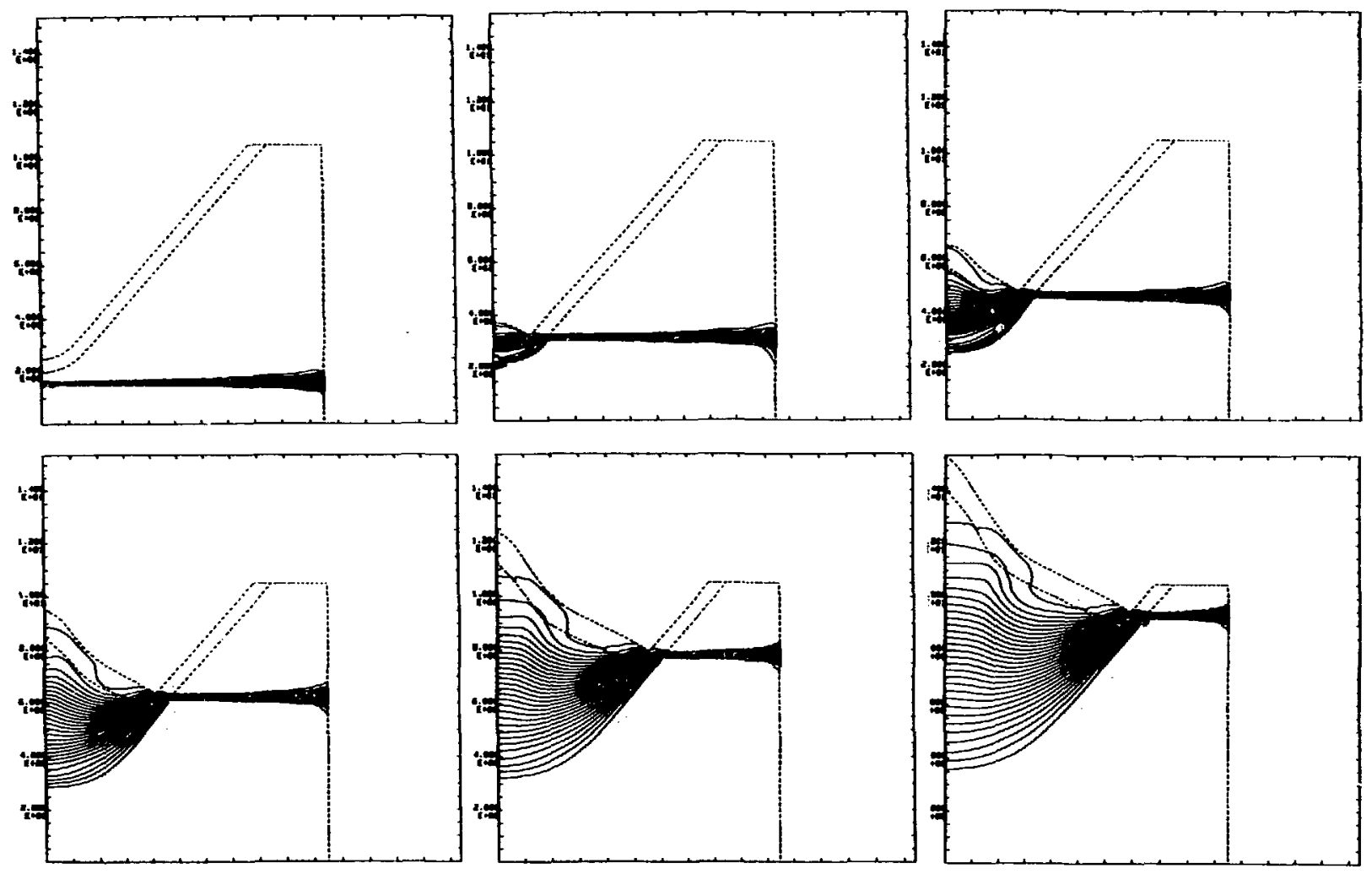

F1g. 44. The evolution of shock structure as an incident shock $(M=3.11)$ passes over a deformable conical shell with shell density equal to 0.5 .

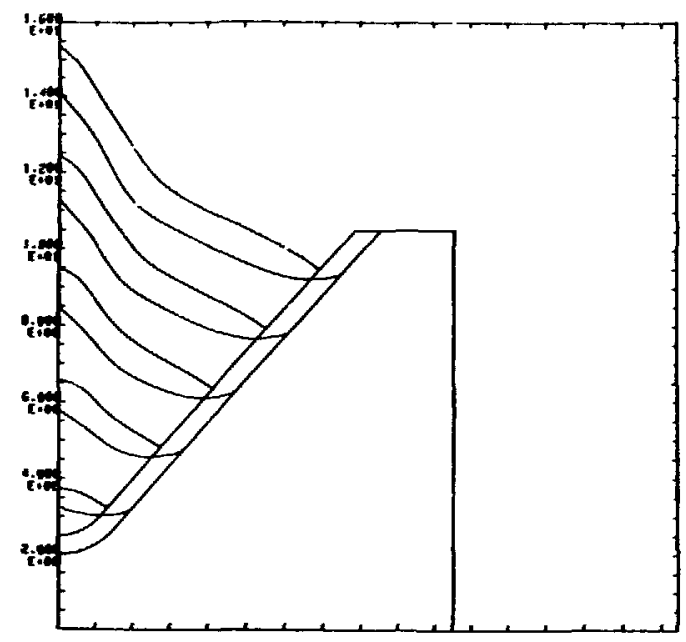

F1g. 45. Shell deformation for the problem of Fig. 44 at the same times as in that figure.

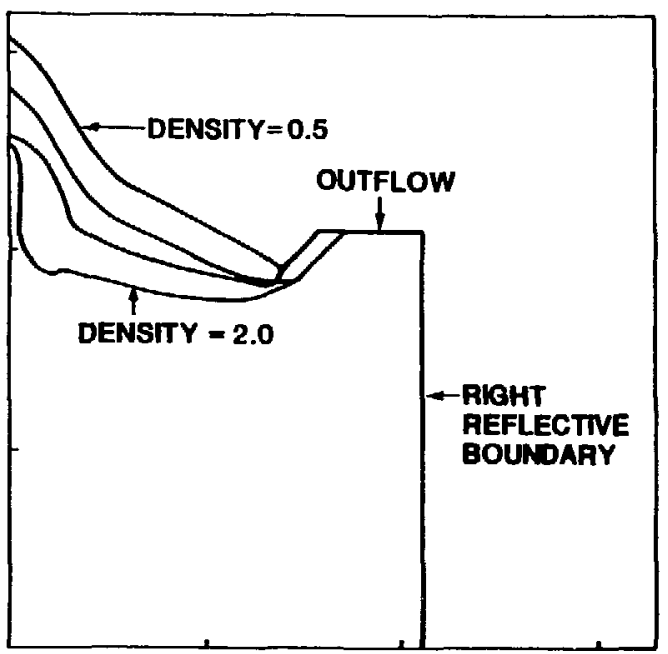

F1g. 46. A comparison of she11 configuration at a late time for the two cases of Figs. 42 and 44. 
For our next calculation we specify the shell density to be equal to 5.0 . We keep ail the other input and inftial conditions the same as in the previous problem. Figure 47 shows the evolution of shock structure at various times. The features in this figure are quite simflar to those of Fig. 42. In this example the transmitted shock moves slower through the deformable conical shell because of the larger density in the she11. Also the speed at which the tip of the conIcal shell moves up the left axis is much slower than In the previous two examples, being just slightly faster than the velocity of the incident shock. Figure 48 shows the evolution of the material configuration. Figure 49 shows the late time mesh configuration. A close examination shows that the mesh has become tangled, with cell edges crossing over each other. This complication caused our calculation to terminate. This difficulty can be corrected by changing some of the rezone parameters. In this calculation we specified an equal spacing of nodes along all the boundarles thus causing our difficulty. We speculate that it would be possible to find a better interface rezoning so that the cells would not fold over each other.

In the final calculation of this study the shell density is 10.0. Figure 50 shows shock evolution and shell deformation at various times. Since the density of the shell is even larger in this calculation, we find that the transmitted shock moves through the shell more slowly than in the previous calculation. The velocity of the tip is in this case only 0.94 times the velocity of the incident shock. Figure 51 shows the shell configuration at the same times as In Fig. 50 .

\section{QUESTIONS AND DISGREPANCIES}

In examining the results of the calculations discussed in this report, we observed a few that seemed questionable in comparison with experimental data, analytical results, or our intultive projection regarding the expected behavior. In this section, we attempt to resolve some of these matters or at least to present plausible explanations. These matters are most appropriately discussed in relation to the figures in which they appear. 

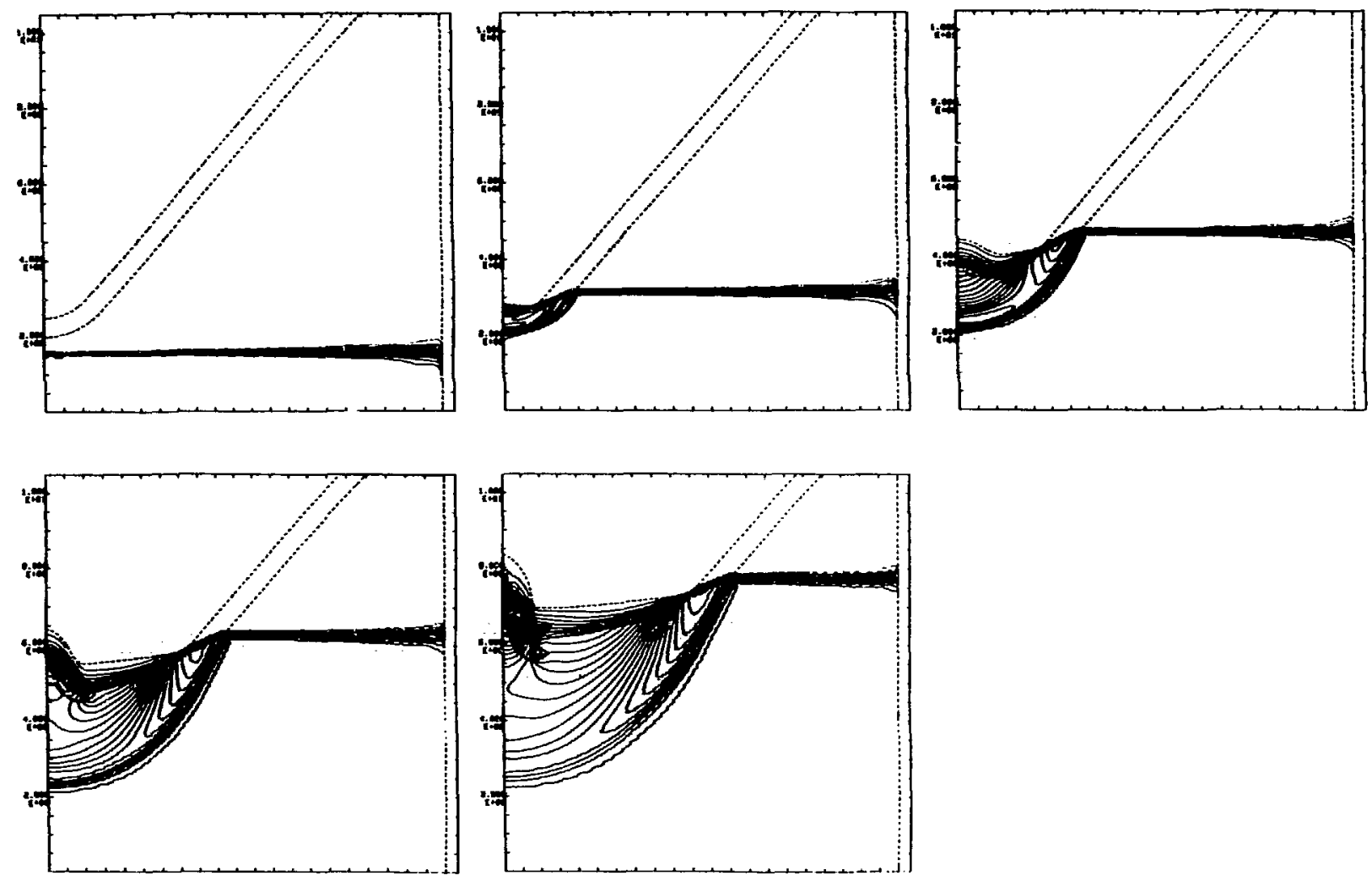

Fig. 47. The evolution of shock structure as an incident shock $(M=3.11)$ passes over a deformable conical shell with shell density equal to 5.0.

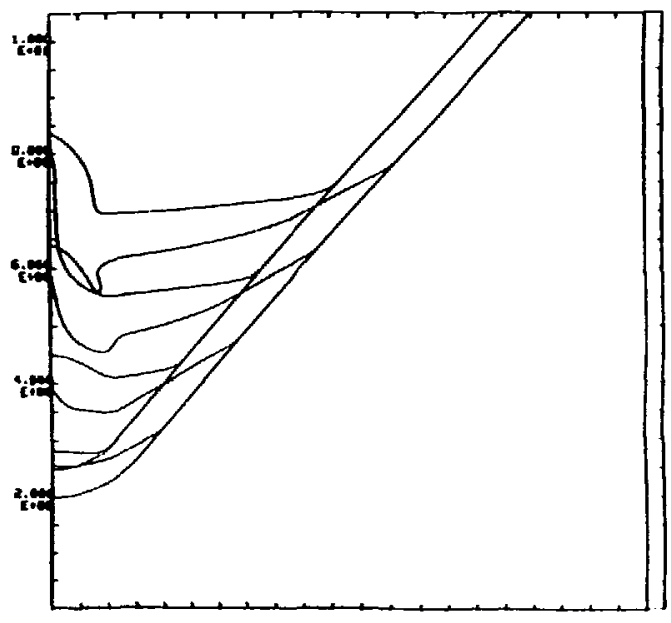

F1g. 48. Shell deformation for the problem of Fig. 47 at the same times as in that figure.

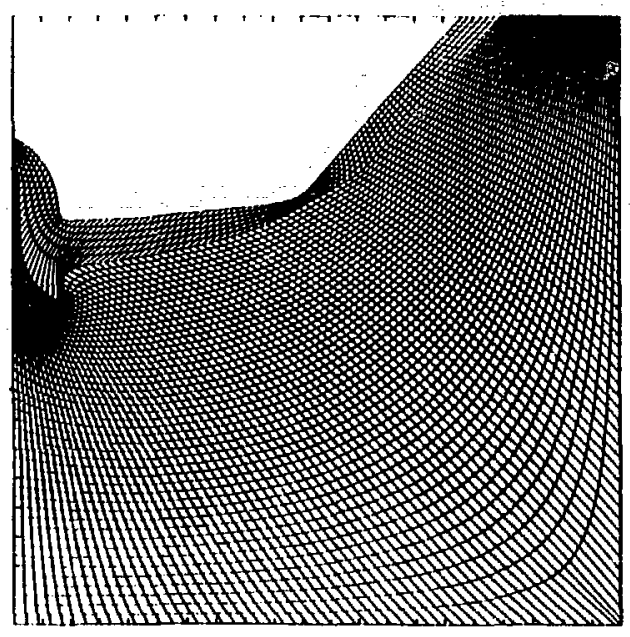

Fig. 49. Late time mesh configuration for conical she11 with ohell density equal 5.0 . 

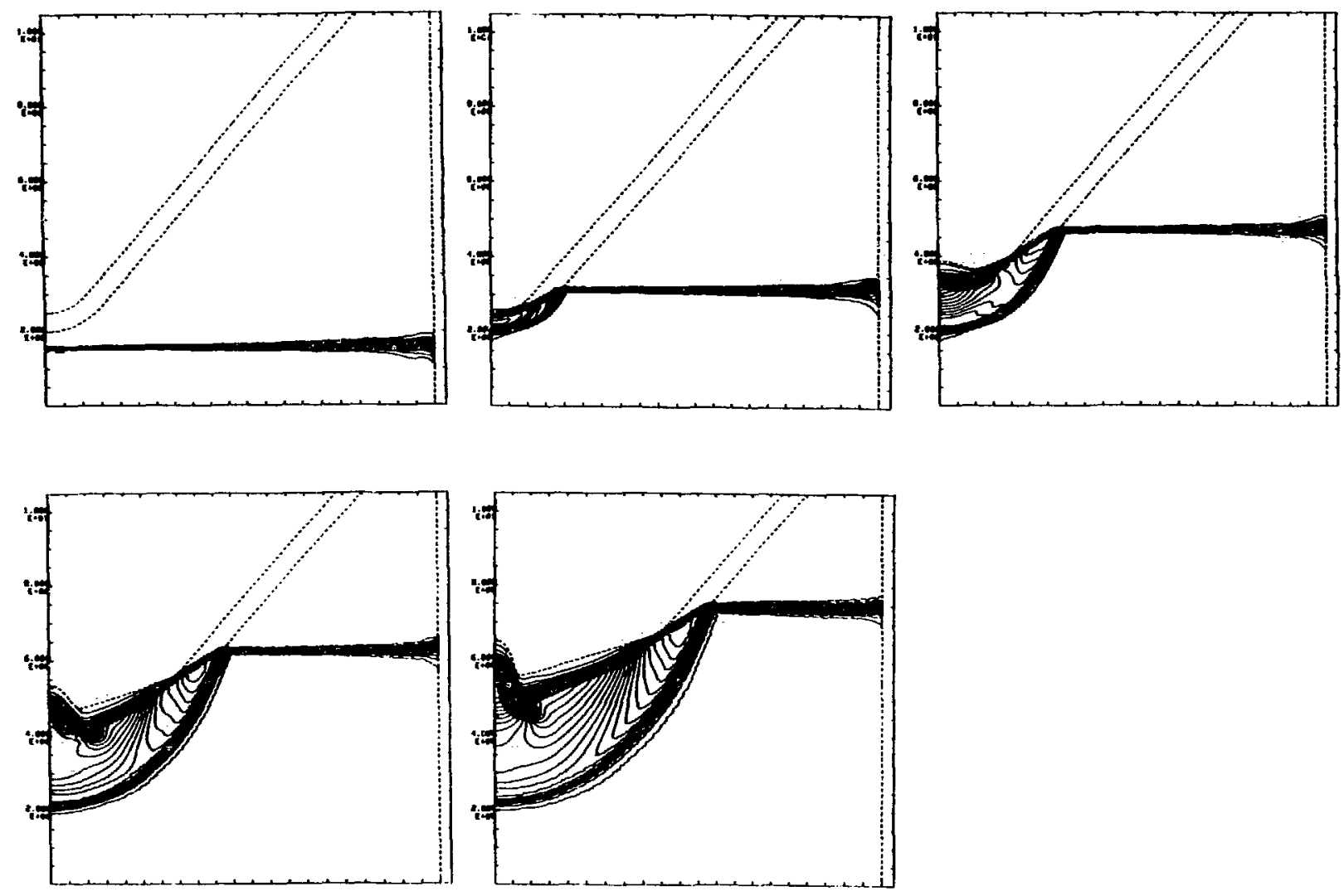

Fig. 50. The evolution of shock structure as an incident shock $(M=3.11)$ passes over a deformable conical shell with shell density equal to 10.0 .

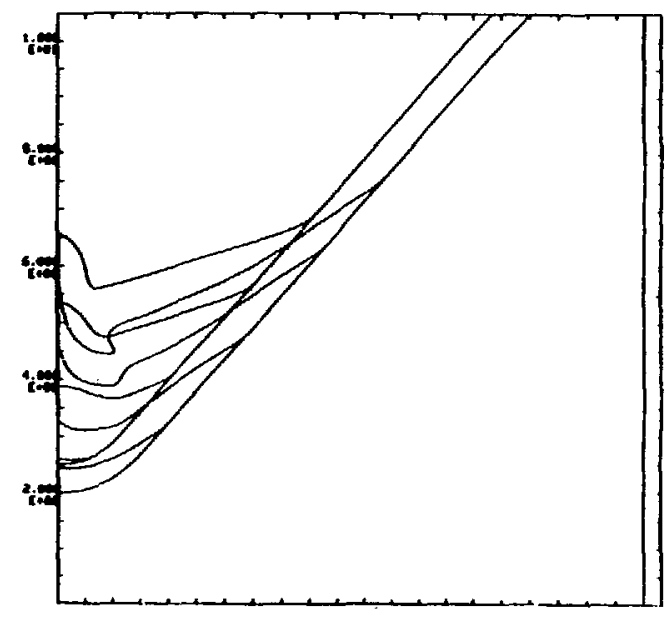

F1g. 51. She11 deformation for the problem of Fig. 50 at the same times as in that figure. 


\section{A. Discrepancles of Figure 4}

There are two matters to discuss relative to FIg. 4. The first concern is the occurrence of two computed pressures at the Incidence angle of 35 degrees. The larger of these agrees well with both the experimental data of Hellig and Reichenbach [2] and with the analytical single shock theory, the solid line in the figure. The smaller, however, 11 es well below the single shock pressure, and appears to be an extension of the double-shock dara and theory to an Incidence angle that is sma11 compared to those that might ordinarily be expected. Heilig and Relchenbach suggest that the Incldence angle of 40 degrees is the smallest for which Mach reflection should be observed. As depicted in Fig. 5, our calculation Indicates a very short Mach stem for this set of circumstances; Indeed, the figure shows that the complex double Mach stem structure is visible at this angle and Mach number. A cursory perusal of avallable 11terature suggests, however, that the Mach reflection In these clrcumstances may well be valid. Alternatively, it is possible that the degree of resolution used for this calculation resolves the dilemma of regular versus Mach reflection towards the latter with a slightly nonphysical bias.

The second 1ssue regarding Fig. 4 concerns the pressures at a 40 degree angle of Incidence where espectally the larger of the two calculational pressure jumps is low in comparison to both the experimental data and the theoretical curve. In this regard, it is relevant to observe that the experimental measurements required special interpretation in the immediate vicinity of this angle, where the theoretical curve shows a very sharp upswing towards singularity. The data polnts marked by an $x$ with error bars were obtalned from measurements of the observed angle of reflection converted to pressure fump by means of a form of Sne11's law. This same type of discrepancy is also observed in Figs. 7 and 9. In our calculations, the pressure behind the leading Mach stem shows a very narrow spike as illustrated In Fig. 52(b), which plots the pressure as a function of position along the wedge at a late time for the clrcumstances of Fig. 7 at an inc1dence angle of 55 degrees. Th1s sharpness of the pressure sptke ls observed in most of our calculations for angles of incidence down to the value at which the single shock singularity occurs. Experimentaliy, this sharpness may have precluded accurate peak pressure measurements due to finite response time of the transducers. In calculations, the accurate determination of peak pressure is impeded by the resolution of the mesh. With self-similar behavior for the solution as it progresses along the wedge through a mesh with unchanging resolution, 
the peak calculated pressure is expected to rise asymptotically to its correct value and this progression is indeed observed in our results. The points shown In the vicinity of the singularicy in Figs. 4, 7, and 9 are low but rising; computer time limitations, however, prevent our extension of the calculation to the ultimate value at this angle.

B. Discrepancieg of Figure 7

Another point of concern regarding Fig. 7 is the relationship between the experimental points and the theoretical curve at angle of incidence greater than 46 degrees. The comments regarding the experimental and calculational points at 46 degrees were discussed in relation to Fig. 4, above. In that figure, the experimental data agreed with the theoretical curve at large angles of Incidence; In FIg. 7, however, there is a discrepancy at those large angles. The calculations furnish a very plausible explanation for this contrast. For the calculatlons shown in FIg. 4, the pressure profiles along the wedge have relatively broad maxima like those of the regular reflection profile in Fig. 52a. For the case of Fig. 7, the profiles are very sharp at all angles above the singularity angle, like those of Fig. 52b. Let us alssume that the experimental transducers responded with a finite relaxation time. Running our calculation to late enough times for resolution of the asymptotic pressure spike enables us to read the pressure at any desired finite time behind that sfike. Assuming spacial delays, $\Delta C$, ranging from 0.5 to 1.2 in vertical calcularional distance units, we can plot the calculated pressure and compare with the experimental data. With $\Delta C$ equal to zero our calculated results are very close to the theoretical curve. With $\Delta C$ equal to 0.6 the calculations match the experimental results quite nicely. KnowIng the actual shock speed in the experiments and the correspondence between experimental and calculational length scales we can transform the observed value of $\Delta C$ to an estimated transducer response time, which in this case 18 approximately $1 \times 10^{-4}$ seconds.

\section{Discrepancies of Figure 17}

Th1s figure shows two discrepancies of interest. One of them is the departure of both the experimental and calculational points from the theoretical curve, which in particular exhibits a sharp rise at 40 degrees. The theoretical curve was derived by considering the pressure fump on a wedge at each of a succession of wedge angles and assuming that the same pressure jump would occur as the shock passed around the cylinder. It is clear that the transient behavior of the dynamica near the intersection point prevents the flow fleld from locally 


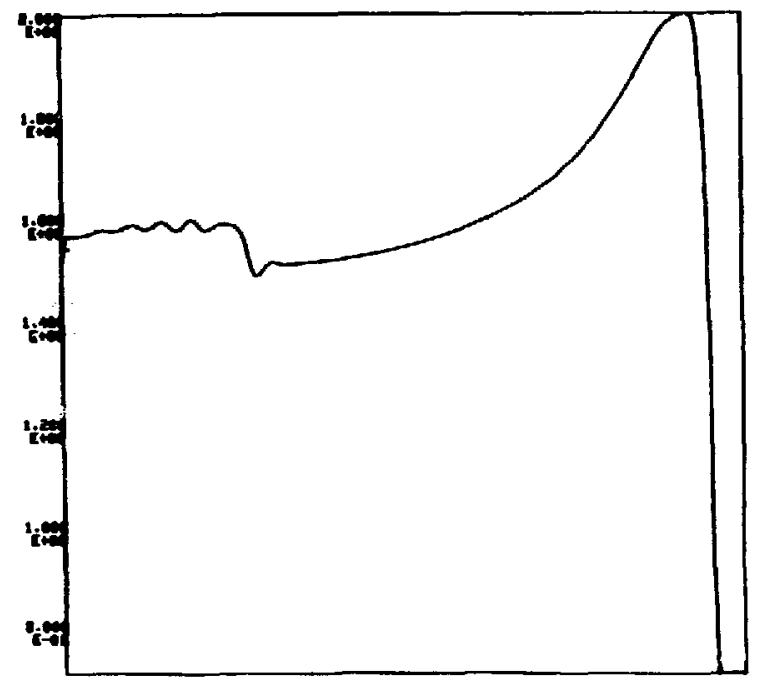

Fig. $52(a)$

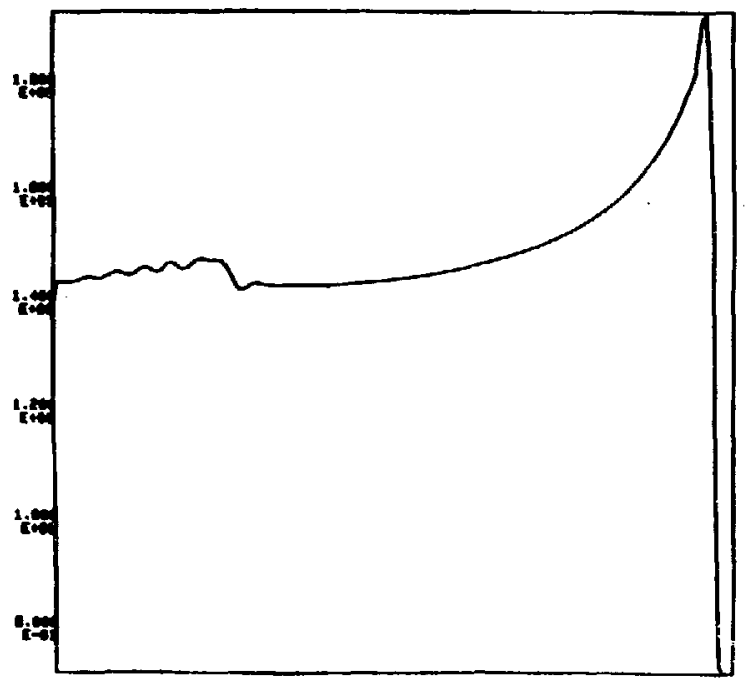

F1g. $52(\mathrm{~b})$

Fig. 52. A comparison of the pressure profiles along the wedge for two different wedge angles and $M=1.26$. (a) shows a broad maxima for regular reflection and (b) shows a narrow splke for simple Mach reflection.

equilibrating to the wedge-theory result for angles greater than about 30 degrees or so. A more important discrepancy in this figure is the disagreement between the experimental results of Hellig and Relchenbach and our calculated results. The experimental data lack error bars but the possible magnitude of the errors may be indicated by the scatter and in particular by the point at 30 degrees. It is not likely, however, that the discrepancy at larger angles can be explained by experimental error. At very large angles, pressure-spike sharpness might preclude transducer resolution, but at those angles (greater than 50 degrees) Hel11g and Relchenbach simply present no data. We are thus at a 108 so explain the observed discrepancy. Two comments, however, may be relevant. One refers to Fig. 19 In which the same discrepancy is observed at a larger Mach number. At angles greater than 60 degrees that figure shows two pressures at each angle, the lower one beling a precursor and the upper derivad from pressures at the knee of a curve like that shown In Fig * 20. This complex structure for the pressure profile at its leading edge lends slight ambiguity to the relationship between experimental and calculational interpretations. The experiments niay be picking up a relative1y low signal for the initial rise at the transducer. Our calculational interpretation of the pressure at the curve's knee may in contrast be too high a representation of what we would expect the experiments to show. It is interesting 
to note In Fig. 27, that allowance for even a slight deformation of the Interface brings the calculational results down toward the experimental values.

\section{CONCLUSION}

These CAVEAT results provide useful and valuable information about the code. Our study of the interaction of shocks in air with rigid wedges, cylinders, and spheres, and with deformable cylindrical, spherical, and conical shells demonstrates considerable accuracy in the CAVEAT code and 11lustrates a variety of avallable options. To demonstrate these features, we have compared our computational results with the experimental data presented by Hellig and Relchenbach [2] and with various theoretical and analytical results. Comparing density contour plots generated from our computational data with Shadow-Schlieren photographs [2] and Interferograms presented by Ben-Dor and Glass [3] we see that the complex shock structures generated from our computational results are very simflar to those of the experiments. We find that our computational results show very good detall of the shock structures and in some cases we see better detall about the shock structure in our computational results then the photographs of the actual experiments provide. In general the numerical calculations almost always produce a wealth of data regarding the dynamical processes far greater than is obtalnable by the performance of an experiment. For example each of the calculations presented In this report gives a complete profile of the changing temperature flelds and velocity vectors throughout both of the interacting materials.

CAVEAT can handle large differences in density between two adjacent materials. When we simulate an almost rigld cylinder or sphere by making the density in the shell region large we get results in close agreement to the rigid cylinder and sphere cases. The study also provides useful information about the adaptive mesh technique implemented in the CAVEAT code. We show that the code is able to handle large deformations as a result of its adaptive rezone capability.

\section{ACRTOWLEDGMTHTS}

I am extremely grateful to Franc1s H. Harlow for hls extensive discussions and technical advice, without which this report would not have been possible.

Discussions and help from Norm Johnson, Frank Addessio, and Bryan Kashiwa are very much appreclated.

I am also especially grateful to Adrienne Hayes for her work in the editing, layout, and design of this report, and to the entire CAVEAT team for their support. 


\section{REFBRENCES}

1. Frank L. Addessio, et al., "CAVEAT: A Computer Code for Fluid Dynamics Problems with Large Distortion and Internal Slip," Los Alamos National Laboratory report LA-10613-MS (February 1986).

2. W. Hellig and $H$. Relchenbach, "Model Test Investigation of Shock Wave Reflection with the Ald of High Speed Shadow-Schlieren Visualization and Pressure Gauges," 16th International Congress on High Speed Photography and Photonics (Strasbourg 1984), M. Andre, M. Hugenschmidt, Editors, Proc. SPIE 491, 583-591 (1985).

3. G. Ben-Dor and I. I. Glass, "Domains and boundarles of non-stationary oblique shock-wave reflexions. 1. Dlatomlc gas," Journal of Fluld Mechanics 92, 459-496 (1979).

4. Francls H. Harlow and Anthony A. Amsden, "Fluid Dynamics," Los Alamos Sclentific Laboratory report LA-4700 (June 1971). 